\title{
Altered motor, anxiety-related and attentional task performance at baseline associate with multiple gene copies of the vesicular acetylcholine transporter and related protein overexpression in ChAT::Cre+ rats
}

\author{
Craig P. Mantanona ${ }^{1} \cdot$ Johan Alsiö $^{2} \cdot$ Joanna L. Elson $^{3} \cdot$ Beth M. Fisher $^{2} \cdot$ Jeffrey W. Dalley $^{2} \cdot$ Timothy Bussey $^{2,4}$. \\ Ilse S. Pienaar ${ }^{5}$
}

Received: 9 May 2019 / Accepted: 3 September 2019 / Published online: 10 September 2019

(c) The Author(s) 2019

\begin{abstract}
Transgenic rodents expressing Cre recombinase cell specifically are used for exploring mechanisms regulating behavior, including those mediated by cholinergic signaling. However, it was recently reported that transgenic mice overexpressing a bacterial artificial chromosome containing choline acetyltransferase (ChAT) gene, for synthesizing the neurotransmitter acetylcholine, present with multiple vesicular acetylcholine transporter (VAChT) gene copies, resulting in altered cholinergic tone and accompanying behavioral abnormalities. Since ChAT::Cre+ rats, used increasingly for understanding the biological basis of CNS disorders, utilize the mouse ChAT promotor to control Cre recombinase expression, we assessed for similar genotypical and phenotypical differences in such rats compared to wild-type siblings. The rats were assessed for mouse VAChT copy number, VAChT protein expression levels and for sustained attention, response control and anxiety. Rats were also subjected to a contextual fear conditioning paradigm using an unconditional fear-inducing stimulus (electrical foot shocks), with blood samples taken at baseline, the fear acquisition phase and retention testing, for measuring blood plasma markers of hypothalamic-pituitary-adrenal gland (HPA)-axis activity. ChAT::Cre+ rats expressed multiple mouse VAChT gene copies, resulting in significantly higher VAChT protein expression, revealed anxiolytic behavior, hyperlocomotion and deficits in tasks requiring sustained attention. The HPA-axis was intact, with unaltered circulatory levels of acute stressinduced corticosterone, leptin and glucose. Our findings, therefore, reveal that in ChAT::Cre+ rats, VAChT overexpression associates with significant alterations of certain cognitive, motor and affective functions. Although highly useful as an experimental tool, it is essential to consider the potential effects of altered cholinergic transmission on baseline behavior in ChAT::Cre rats.
\end{abstract}

Keywords ChAT::Cre rats · Cre-recombinase · 5-CSRTT · HPA endocrine axis · Touchscreen-based behavioral testing · Vesicular acetylcholine transporter

Timothy Bussey and Ilse S. Pienaar are the equal senior contributors.

$\begin{array}{ll}\text { Abbreviations } \\ \text { ACh } & \text { Acetylcholine } \\ \text { AChE } & \text { Acetylcholinesterase } \\ \text { AD } & \text { Alzheimer's disease } \\ \text { ADHD } & \text { Attention deficit/hyperactivity disorder }\end{array}$

Ilse S. Pienaar

i.s.pienaar@sussex.ac.uk

1 Faculty of Health and Life Sciences, Northumbria University, Newcastle upon Tyne, UK

2 Department of Psychology, The Behavioral and Clinical Neuroscience Institute, University of Cambridge, Downing Street, Cambridge, UK
3 Institute of Genetic Medicine, Newcastle University, Newcastle upon Tyne, UK

4 Department of Physiology and Pharmacology, Robarts Research Institute, University of Western Ontario, London, ON, Canada

5 School of Life Sciences, University of Sussex, Falmer BN1 9PH, UK 


$\begin{array}{ll}\text { BAC } & \text { Bacterial artificial chromosome } \\ \text { BSA } & \text { Bovine serum albumin } \\ \text { 5-CSRTT } & \text { Five-choice serial reaction time task } \\ \text { ChR2 } & \text { Channelrhodopsin-2 } \\ \text { ChAT } & \text { Choline acetyltransferase } \\ \text { CFCT } & \text { Contextual fear conditioning task } \\ \text { DREADD } & \text { Designer receptors exclusively activated by } \\ & \text { designer drugs } \\ \text { DAT } & \text { Dopamine transporter } \\ \text { EPM } & \text { Elevated plus maze } \\ \text { eYFP } & \text { Enhanced yellow fluorescent protein } \\ \text { EtOH } & \text { Ethanol } \\ \text { CHT1 } & \text { High-affinity choline transporter } \\ \text { HSD } & \text { Honestly significant difference } \\ \text { HPA } & \text { Hypothalamic-pituitary-adrenal } \\ \text { ITI } & \text { Intertrial interval } \\ \text { LSD } & \text { Least significant difference } \\ \text { LDB } & \text { Light/dark box } \\ \text { LH } & \text { Limited hold } \\ \text { NS } & \text { Non-significant } \\ \text { NBMc } & \text { Nucleus basalis magnocellularis } \\ \text { NBM } & \text { Nucleus basalis of Meynert } \\ \text { OF } & \text { Open field } \\ \text { PD } & \text { Parkinson's disease } \\ \text { PPN } & \text { Pedunculopontine nucleus } \\ \text { PCR } & \text { Polymerase chain reaction } \\ \text { PVDF } & \text { Polyvinylidene difluoride } \\ \text { PFC } & \text { Prefrontal cortex } \\ \text { qPCR } & \text { Quantitative PCR } \\ \text { RIPA } & \text { Radioimmunoprecipitation assay } \\ \text { RT-PCR } & \text { Real-time PCR } \\ \text { RRID } & \text { Research resource identifier } \\ \text { RT } & \text { Room temperature } \\ \text { SLC5A7 } & \text { Solute carrier family 5 member 7 } \\ \text { SD } & \text { Stimulus duration } \\ \text { TBS-T } & \text { Tris-buffered saline-Tween 20 } \\ \text { TH } & \text { Tyrosine hydroxylase } \\ \text { VAChT } & \text { Vesicular acetylcholine transporter } \\ \text { Wt. } & \text { Wild-type } \\ & \end{array}$

\section{Introduction}

Central cholinergic signaling is required for regulating aspects of memory, motivation and mood. On the other hand, cholinergic deficiency contributes to the neuropsychiatric symptoms associated with Alzheimer's disease (AD), with cholinomimetic therapies that may ameliorate such symptoms (Felder et al. 2018). In addition, although the main motor deficits characterizing Parkinson's disease (PD) patients stem from the near total loss of midbrain dopaminergic neurons, the concomitant loss and/or dysfunction of cholinergic neurons is believed to underlie such patients' cognitive decline (Perez-Lloret and Barrantes 2016; Bury et al. 2017). Hyperactivity of brain cholinergic systems could also underlie the cognitive disturbances observed in patients with affective disorders (Saricicek et al. 2012; Mineur et al. 2013), while additional evidence for this derives from clinical and preclinical studies which revealed that cholinergic receptor blockers can induce antidepressant-like responses (Furey and Drevets 2006). Furthermore, increasing data from clinical pharmacology, neuroimaging and postmortem studies implicates the cholinergic system in the pathogenesis of schizophrenia (Sarter et al. 2012). Conversely, growing evidence suggest that pharmacologically increasing cholinergic signaling could improve several symptom clusters, including visual hallucinations, in schizophrenia patients (Foster et al. 2012). However, despite the accumulating evidence in support of cholinergic perturbations as an etiological factor in several neuropsychiatric human diseases, patients remain mostly unresponsive to current cholinergicbased pharmacological therapies. This highlights a need for establishing appropriate mammalian models for understanding the cholinergic roots of these disorders and for in vivo validation of candidate therapeutics that are potentially more effective treatments, by specifically correcting the relevant pathophysiological mechanisms.

For modeling human disease, rats offer several distinct advantages over mice and other organisms. Benefits include increased spatial resolution due to rats' larger sized heads, which provides practical advantages for performing surgical procedures, including intracerebral cannula implantation for localized drug infusion into a specific brain region, to determine the role of this region in a particular behavioral phenotype (Kokare et al. 2011). Proportionally, larger brain substructure sizes also lends itself to more precise implantation of microdialysis probes for sampling neurotransmitter concentrations, as the cannula causes less damage by affecting smaller regions. Although mice continue to offer distinct advantages over rats for precise targeting of gene expression to defined neuronal populations (Taniguchi et al. 2011; Madisen et al. 2012; Gerfen et al. 2013), tremendous strides made in developing a toolbox of transgenic rat lines have allowed for unprecedented interrogation of cell type-specific neural populations and -circuits. Such experimental strides are permitted by engineering cell-specific targeting in a Cre recombinase-dependent manner, along with advances made in engineered viral vectors. These transgenic rat technologies can be combined with opsin- and Designer Receptors Exclusively Activated by Designer Drugs (DREADDs), for untangling the neural substrates of a wide plethora of behaviors; these are beginning to be tapped in research studies (e.g., Sharma and Pienaar 2014, 2018).

Choline acetyltransferase (ChAT)::Cre+ transgenic rats, utilizing bacterial artificial chromosome (BAC) recombineering for introducing a transgene of interest under control of 
cholinergic neuronal-specific promoter elements (Witten et al. 2011) were generated to genetically restrict expression of Cre recombinase in cholinergic neurons. As an additional genetic element, the BAC is inserted within the genome, sometimes in multiple copies, with the original ChAT locus that remains intact.

Such rats are extremely useful in promoting understanding of cholinergic circuits (Aldrin-Kirk et al. 2018; Dautan et al. 2014, 2016; Gielow and Záborszky 2017) including their role in motor function and reward-related behaviors during both homeostatic and disease processes (Pienaar et al. 2015; Dautan et al. 2016; Xiao et al. 2016). The generation of these transgenic rats followed on from work using transgenic mice was designed to encode channelrhodopsin-2 (ChR2) protein under the control of the ChAT promoter (Heintz 2001). This design largely ignores the tandem gene arrangement for the cholinergic gene locus (Prado et al. 2017), where the gene for the vesicular acetylcholine transporter is nested within the first intron of the ChAT gene. Evidence is accumulating that in ChAT::Cre+ rodents, altered expression of the gene encoding for the vesicular acetylcholine transporter (VAChT), which serves as a key molecular component of acetylcholine (ACh) release by loading $\mathrm{ACh}$ molecules into the synaptic vesicles of cholinergic synapses, could modify baseline cholinergic tone. This modification could provide a mechanistic basis for changing aspects such as locomotion, operant conditioning, spatial and working memory, attention, as well as intravenous nicotine self-administration behaviors, as had been reported for ChAT-ChR2-eYFP BAC transgenic mice, compared to wildtype (Wt/Wt) and hemizygous (Tg/Wt) littermates (Kolisnyk et al. 2013; Sugita et al. 2016; Chen et al. 2018; Janickova et al. 2017).

Here, we determined the mRNA and protein expression levels of VAChT in ChAT::Cre+ rats compared to Wt (ChAT::Cre-) ones, to gain mechanistic understanding as to the influence exerted by altered expression of cholinergic signaling factors in performance in the five-choice serial reaction time task (5-CSRTT), a well-validated measure of attention and impulsivity (Robbins 2002). We further conducted motor and anxiety profiling in the transgenic rats compared to their Wt littermates, using a range of well-validated behavior tests. Due to compelling evidence that the cholinergic system plays a role in stress and, specifically in hypothalamic-pituitary-adrenal gland (HPA)-axis activation and regulation, we also determined whether ChAT::Cre+ rats manifest a dysregulation of this endocrine control system (Paul et al. 2015).

\section{Materials and methods}

\section{Animals}

The animal experiments were approved by an ethics panel at Northumbria University (Ref: BMS39UNNGB2015) and were performed in accordance with the Animals (Scientific Procedures) Act, 1986 (UK) for the care and use of experimental animals as well as the European Communities Council Directive (2010/63/EEC). All rats were examined weekly by a veterinarian and constantly monitored by experienced animal technicians for signs of pain or distress. Long-Evans Wt rats [Charles River, Kent, UK; Research Resource Identifier (RRID): RGD_2308852] were bred with hemizygous Long-Evans ChAT::Cre rats (Missouri Mutant Mouse Regional Resource Centre, University of Missouri, USA; RRID: RGD_10401204); both providers supplied up-to-date authentication reports. Once weaned, ear clippings were taken from each pup and analyzed for the Cre recombinase gene using polymerase chain reaction (PCR). The primers used were as described by Witten et al. (2011) and consisted of AAG AACCTGATGGACATGTTCAGGGATCG (forward) and CCACCGTCAGTACGTGAGATATCTTTAACC (reverse), which produced a positive band corresponding to 600 base pairs. The PCR cycling conditions were similar to those described by Witten et al. (2011).

Only mature male rats (weight: $270-310 \mathrm{~g}$ ) were used in the experiments to minimize gender and hormonal influences on behavior (Keeley et al. 2015). As an additional control condition for analyzing mouse VAChT gene copy number, brain tissue was also collected from male littermate Wt control mice (weight: $25-30 \mathrm{~g}$, on the C57BL/6 background, received from Charles River, UK).

Animals were housed in groups of 3-4 per cage in polypropylene cages measuring $56 \times 38 \times 22 \mathrm{~cm}(\mathrm{RC} 2 \mathrm{~F}$; North Kent Plastics Cages, Coalville, UK) within an animal care facility at the University of Cambridge, in a temperature $\left(23{ }^{\circ} \mathrm{C}\right)$ and humidity $(45-65 \%)$ controlled room with a reversed light/dark cycle (lights off between 7:00 and 19:00 h). The behavioral experiments were carried out at the same time each day, during the dark period which represents the rats' nocturnal phase of the circadian cycle, during which they experience their most active, inquisitive and responsive period (Gorka and Adamik 1993). As the rats were maintained on a reverse light/dark cycle, this enabled us to conveniently test the rats during their activity phase.

One group was used for testing in the 5-CSRTT (ChAT::Cre+, $n=13$; ChAT::Cre-, $n=8$ ), whilst the second group was subjected to anxiety-profiling tasks, consisting of the open field (OF) arena, elevated plus maze 
(EPM), and light/dark box (LDB) (ChAT::Cre+, $n=8$; ChAT::Cre- $n=10$ ). Finally, some of the rats (that had been subjected to either the 5-CSRTT or anxiety testing) were subjected to fear conditioning testing followed by blood/tissue analysis (ChAT::Cre+, $n=5$; ChAT::Cre-, $n=8$ ). During 5-CSRTT training, the animals had their weight monitored weekly and maintained at $85-95 \%$ of their free feeding body weight. During this period, the animals received $18 \mathrm{~g}$ of laboratory pellet chow once a day with ad libitum access to water. Animals used for anxiety profile tasks and fear conditioning followed by blood/tissue analysis had ad libitum access to food and water. OF, EPM, LDB, fear conditioning testing and blood sampling occurred between 14:00 and 17:00. The order of behavioral assessment was anxiety profile tasks (OF, EPM and LDB) followed by the contextual fear conditioning task (CFCT) and then blood sampling. No sample calculation was performed to estimate the number of rats used in each experimental group.

\section{Behavioral procedures}

Blind scoring of the rats' behavioral test performance was performed using automated computer software. Additionally, the experimenter was blind to genotype during ratderived sample processing. For each behavioral test, rats were tested in a counterbalanced order with regard to genotype. Following testing of each animal, the apparatus was thoroughly cleaned with $70 \%$ ethanol $(\mathrm{EtOH})$ to eliminate the residual odors and traces of the previously tested animal.

\section{Open field testing}

All behavioral procedures were conducted in a dark (visibility was $5 \mathrm{~m}$ ), soundproofed room. For measuring behavior in the OF arena, rats were placed in the center of a circular field of $900 \mathrm{~cm}$ (diameter) $\times 38.5 \mathrm{~cm}$ (height). The apparatus consisted of an inner field (625 $\mathrm{cm}$ in diameter) surrounded by an outer field (375 cm from inner field edge to field's edge). Room temperature (RT) and lighting were identical to that used for testing in the EPM (see below). Each rat was recorded for 5 min using a Yi Action Camera (Yi Technology, Seattle, USA) connected to a personal computer, with subsequent videos analyzed using ANY-maze software (Stoelting ANY-maze, Wood Dale, USA). Parameters were time spent in inner field ( $\%$ of time), number of entries into inner field, distance traveled (m), number of bouts and time spent grooming $(\mathrm{s})$, number of bouts and time spent rearing $(\mathrm{s})$, and average speed $(\mathrm{cm} / \mathrm{s})$.

An animal was recorded to have entered either the inneror outer field of the test area when all four paws had been placed inside either zone. The protocol stipulated that a rat would be excluded from analysis if a noise or other disruption occurred in the test environment, as a likely influencer of the rat's behavior.

\section{Elevated plus maze testing}

At least 7 days after conducting OF testing on the rats, the rats' exploratory behavior in the EPM was recorded using the same recording equipment described above. The EPM was constructed from black and clear Perspex and consisted of two open arms $(110 \mathrm{~cm}$ long $\times 11 \mathrm{~cm}$ wide $)$ and two closed arms $(110 \mathrm{~cm}$ long $\times 10 \mathrm{~cm}$ wide $\times 40 \mathrm{~cm} \mathrm{high})$, extending from a central platform, which was raised $50 \mathrm{~cm}$ from the ground. The testing room was illuminated evenly with white light (intensity $70 \mathrm{~lx}$ ). The protocol was run as previously described (Walf and Frye 2007). Briefly, rats were placed facing the same open arm in the central platform and exploratory behavior was recorded for $5 \mathrm{~min}$. Animals were excluded from analysis under the following conditions: when a rat fell over the edge of the open arms of the maze and if a noise or other disruption caused the rat to freeze during testing. The parameters analyzed were time spent in open arms (\% of time), number of entries into open arms $(n)$, distance traveled $(m)$, number $(n)$ and time spent grooming $(\mathrm{s})$, number $(n)$ and time spent rearing (s), and average speed $(\mathrm{cm} / \mathrm{s})$. ANY-maze software was used to analyze the videos.

The EPM testing protocol stated that entry into an arm was only scored when all four paws of the animal had entered an arm, while a rat that fell over the edge of the open arms of the maze or froze during testing due to a noise or other disruption would be excluded.

\section{Light/dark box testing}

Rodents prefer dark and enclosed places, as a strategy for reducing the risk of predation (Crawley and Goodwin 1980). With this regard, the LDB assay has been deemed an ethologically relevant measure of anxiety-like behavior in rodents as it assesses approach versus avoidance behavior, baseline vigilance and defensive behaviors (Lezak et al. 2017). Both groups of rats were subjected to an LDB test paradigm at least 7 days after completing testing in the EPM. We utilized an LDB apparatus similar to that described by Bourin and Hascoe (2003), consisting of a plastic box divided into two equally sized partitions: a light-(brightly lit with white walls) and dark (minimally lit with black walls) chamber. The chambers were divided by means of a Perspex partition containing an opening that was large enough for an adult male rat to transverse between the two chambers, with the opening that was covered by a sliding door. All ChAT::Cre+ and ChAT::Cre- rats were tested in the same testing box. Testing was performed in accordance with previously described procedures (Arrant et al. 2013). The test commenced with a rat placed in the dark half of 
the box, with the sliding door that was raised simultaneously. Each animal's behavior was recorded for 5 min using the recording equipment described above. Through the use of ANY-maze software, we analyzed the recordings for the parameters: (1) the latency to enter the light chamber, (2) total time spent in the light chamber and (3) the number of entries into the light chamber.

An entry into either the light or dark chamber was scored when all four paws of the animal had been placed into the respective chamber to be considered an entry. The only exclusion criteria that had been preset were conditions of unforeseen loud noise or other disturbances, for which the protocol stated that a subject would then be removed from the analysis.

\section{Five-choice serial reaction time task}

The 5-CSRTT is a well-validated task for assessment of several cognitive functions and manifesting behaviors including vigilance, impulsivity and compulsivity (Bari et al. 2008). Training and testing took place in Bussey-Saksida Touch System chambers (Lafayette Instrument, Indiana, USA) networked to a computer using 'Whisker' software. Chambers were trapezoidal $[30 \mathrm{~cm}$ (height) $\times 35 \mathrm{~cm}$ (length) $\times 30 \mathrm{~cm}$ front end (width) $\times 25 \mathrm{~cm}$ rear end (width)], with a touchsensitive liquid crystal display flat screen placed at the front end. A food magazine was placed at the rear end, into which $45 \mathrm{mg}$ reward sugar pellets (Sandown Scientific, Middlesex, UK) were dispensed from an external dispenser. Black perspex masks with five evenly spaced square apertures located basally in the rat's immediate field of vision were secured in front of the touch screens. Infrared sensors were located at the front and rear of the chamber.

Briefly, animals were trained to nose-poke into one of five briefly illuminated apertures, spaced randomly. The baseline and training task ended after 30 min or when animals had completed 100 trials. A stimulus duration (SD) of $0.5 \mathrm{~s}$ is standard for 5-CSRTT when assessing rats. However, behavioral output was largely below criteria ( $\geq 70 \%$ accuracy and $\leq 20 \%$ omissions) at an average SD of $0.5 \mathrm{~s}$, so an $\mathrm{SD}$ of $0.6 \mathrm{~s}$ was instead utilized as the baseline level. Four ChAT::Cre+ and two ChAT::Cre- animals were excluded from further testing due to failure to reach final criteria. After establishing that baseline criteria were consistently met, rats were assessed using a varied SD probe $(0.15,0.3$ and $0.6 \mathrm{~s})$. Variables recorded at each session included: (1) correct responses; (2) incorrect responses; (3) accuracy (number of correct responses divided by the total of correct and incorrect responses, expressed as a percentage); (4) omitted responses; (5) premature responses [responses made during intertrial interval (ITI)]; (6) perseverative responses (repeat responses made after a correct response); (7) correct response latency (time from stimulus presentation until a correct response was made); (8) incorrect response latency (time from stimulus presentation until an incorrect response was made); and (9) magazine latency (time to collect reward post-correct response).

Although environmental noise was kept to a minimum, the 5-CSRTT protocol stated that in case of an unexpected noise or other disturbance occurring during 5-CSRTT testing, a rat affected would be removed from analysis.

\section{Animal killing and tissue handling for molecular analyses}

At the end of the behavioral assessments, the rats were killed humanely by exposure to $\mathrm{CO}_{2}$ and the heads were decapitated. The brains were rapidly removed from the skull, immediately followed by bilateral microdissection of the prefrontal cortex (PFC) brain tissue on a prechilled metal plate. All dissection instruments were prechilled in powdered dry ice. The specimens were then flash frozen in liquid nitrogen and stored at $-80^{\circ} \mathrm{C}$ until further use within labeled tubes that were immediately sealed. All steps in the procedure were performed rapidly to avoid RNA and protein degradation.

\section{Contextual fear conditioning task}

The CFCT is a well-validated behavioral assessment measure that is used for exploring the neural substrates involved in emotional learning and memory (Tavote et al. 2015; Kim and Jung 2006). Here, we utilized this Pavlovian conditioning task to monitor HPA-axis functioning in ChAT::Cre+ compared to ChAT::Cre- rats, by measuring levels of blood plasma markers to an acute stressor. Acquisition and retention testing took place in operant conditioning chambers (Med Associates, Vermont, US) measuring $30.5 \mathrm{~cm}$ (length) $\times 32.5 \mathrm{~cm}$ (height) $\times 24.1 \mathrm{~cm}$ (wide), with a plexiglass door and ceiling and metal paneling on the back and on the sides. The floor of the chamber consisted of a metal grid through which a mild electrical shock (115 VAC, $60 \mathrm{~Hz}$; ENV-224AMWN, Med Associates) was delivered. Testing chambers were placed in sound and light attenuating boxes that had been networked to a computer using 'Whisker' software (Campden Instruments Ltd., Loughborough, UK) (Cardinal and Aitken 2010). On the day of training, each rat was placed in the chamber for $3 \mathrm{~min}$, during which time it received no stimulation or cues. At the end of the third minute, rats received $3 \times 1 \mathrm{~s}$ foot shocks of $1 \mathrm{~mA}$ intensity with $60 \mathrm{~s}$ intershock intervals. Animals were removed $30 \mathrm{~s}$ after the final foot shock, which placed acquisition duration at $330 \mathrm{~s}$. Retention testing took place $24 \mathrm{~h}$ later, with each animal being placed in their respective chamber and their behavior recorded for $8 \mathrm{~min}$. Freezing behavior (lack of movement, except for breathing) was analyzed using 
Matlab software (MathWorks, Massachusetts, US). Blood plasma samples were collected from each rat to represent the baseline, acquisition and retention phases of the CFCT, with procedural details provided below.

\section{Blood plasma analysis of corticosterone, leptin and glucose levels}

Blood was taken sublingually under general anesthesia (5\% isoflurane). Baseline samples were collected 2 days prior to initiating the task protocol, at the same time of day that blood would be collected on contextual fear conditioning testing days, which occurred 25 min post-acquisition training and also the same length of time post-retention testing. Approximately, $1 \mathrm{ml}$ samples were taken using ethylenediaminetetraacetic acid (EDTA)-primed vials (Thermo Fisher Scientific, UK), which were then placed on ice and centrifuged at $3000 \times g$ at $4{ }^{\circ} \mathrm{C}$ for $20 \mathrm{~min}$. Plasma was aliquoted into Eppendorf tubes for corticosterone level analysis. Corticosterone (Cat No. ADI-901-097, Enzo Life Sciences, Devon, UK; RRID: AB_2307314) and leptin (Cat No. ADI-900-015A, Enzo Life Sciences, UK) quantification was done using enzyme-linked immunoassay (ELISA) kits with glucose quantification that was done using a colorimetric assay kit (Cat No. 10009582, Cayman Chemical, Michigan, USA), all according to the manufacturer's instructions. The plasma corticosterone and leptin concentration levels are given as $\mathrm{ng} / \mathrm{ml}$, while glucose plasma concentration is given as $\mathrm{mg} / \mathrm{ml}$.

\section{Quantitative RT-PCR}

To examine the copy number of mouse VAChT transgene, we used primers specific for the mouse VAChT gene (VAChTintron forward: GAGAGTACTTTGCCTGGGAGGA and VAChT-intron reverse: GGCCACAGTAAGACCTCC CTTG) and actin primers (actin forward: TATCCTGGC CTCACTGTCCA and actin reverse: AAGGGTGTAAAA CGCAGCTCA), for normalization. Actin primers were able to efficiently amplify both mouse and rat genes. Both primer pairs showed efficiency above $95 \%$ and similar amplified fragment size. We compared amplification of Cre+ rat DNA to Cre- rat DNA and also mouse DNA. Briefly, DNA was isolated from either rat (ChAT::Cre+, $n=5$; ChAT::Cre-, $n$ $=8)$ or mouse $(n=4)$ PFC brain tissue samples that had been pooled according to the genotype, using the phenol-chloroform extraction protocol (Guzman et al. 2011), precipitated with isopropanol and washed twice on $70 \% \mathrm{EtOH}$. The DNA pellet was dissolved in ultra-pure water and concentration was determined based on Nanodrop measurements. PCR reaction was prepared using the Sensifast SYBR Green kit (Cat No. BIO-98020, Bioline, UK). We used a 10- $\mu 1$ total volume, with each primer at concentration of $200 \mathrm{nM}$ and a genomic DNA concentration of $10 \mathrm{ng}$. Technical triplicates were applied and melting curve was checked for each run. Quantitative PCR (qPCR) was performed, using the C1000TM Thermal Cycler, CFX96 Real-Time System (Bio-Rad) at the following settings: step $1: 95^{\circ} \mathrm{C}$ for $5 \mathrm{~min}$; step 2: $95{ }^{\circ} \mathrm{C}$ for $30 \mathrm{~s}$; step $3: 60{ }^{\circ} \mathrm{C}$ for $10 \mathrm{~s}$; step $4: 72{ }^{\circ} \mathrm{C}$ for $10 \mathrm{~s}$; step 5: plate reading. Steps $2-5$ were repeated 40 times. Copy number of mouse VAChT transgene in rats was determined by the $2^{-\Delta \Delta \mathrm{Cq}}$ method (Livak and Schmittgen 2001). The mean $C_{\mathrm{q}}$ of the mouse $V A C h T$ gene and the mean $C_{\mathrm{q}}$ of actin gene in the ChAT::Cre+ and ChAT::Cre- rats were calculated with fold-change that was determined using the $2^{-\Delta \Delta \mathrm{Cq}}$ method. One group was used for testing in the 5-CSRTT (ChAT::Cre+, $n=13$; ChAT::Cre-,$n=8$ ), whilst the second group was subjected to anxiety-profiling tasks, consisting of the OF arena, EPM and light/dark box (LDB) (ChAT::Cre+, $n=8$; ChAT::Cre-, $n=10$ ).

For quantifying VAChT mRNA expression, total RNA was isolated using the Aurum Total RNA Fatty and Fibrous Tissue Kit (Cat no. 7326830, Bio-Rad, Hercules, CA, USA), in accordance with the manufacturer's instructions, using the remainder of the genotype-pooled rat PFC samples (ChAT::Cre+, $n=5$; ChAT::Cre-, $n=7$ ). cDNA was synthesized using a High Capacity cDNA reverse transcription kit (Thermo Fisher Scientific, UK), again following the manufacturer's manual. cDNA was then diluted and the quantitative Real-Time PCR (RT-PCR) reaction was performed on a C1000TM Thermal Cycler, CFX96 Real-Time System (BioRad) using Bioline Sensifast SYBR Green (BIO-98020) using the following PCR cycling conditions: step $1: 95^{\circ} \mathrm{C}$ for $5 \mathrm{~min}$; step 2: $95{ }^{\circ} \mathrm{C}$ for $10 \mathrm{~s}$; step 3: $60{ }^{\circ} \mathrm{C}$ for $10 \mathrm{~s}$; step 4: $72{ }^{\circ} \mathrm{C}$ for $10 \mathrm{~s}$; step 5: plate reading, with steps $2-5$ repeated 40 times. The primer pairs were as follows: mouse VAChT-exon forward: CCCTTTTGATGGCTGTGA, mouse VAChT-exon reverse: GGGCTAGGGTACTCATTAGA. Rat $\beta$-actin was used as reference gene for data normalization, as the actin primer can detect both rat and mice mRNA and genomic DNA. For detecting actin, the following primer pairs were used: rat $\beta$-actin forward: TATCCTGGCCTC ACTGTCCA, rat actin reverse: AAGGGTGTAAAACGC AGCTCA. Technical triplicates were applied and melting curve was checked, as a standard procedure for each plate.

\section{Western immunoblotting}

The PFC rat brain tissue samples, pooled according to genotype, were lysed using radioimmunoprecipitation assay (RIPA) lysis buffer, followed by separation of proteins on 10\% SDS-PAGE (sodium dodecyl sulfate-polyacrylamide) gels. Proteins were transferred to Immobilon$\mathrm{P}$ polyvinylidene difluoride (PVDF) membranes (Cat No. IPVH00010, Millipore, UK) using a semi-dry transfer apparatus (Trans-Blot Turbo ${ }^{\mathrm{TM}}$, Bio-Rad) run at $20 \mathrm{~V}$ for $1 \mathrm{~h}$. The 
membrane was then blocked in $5 \%(\mathrm{w} / \mathrm{v})$ powdered skimmed milk in Tris-buffered saline/ $0.1 \%$ Tween 20 (TBS-T) for $1 \mathrm{~h}$, followed by incubation overnight at $4{ }^{\circ} \mathrm{C}$ with a primary antibody raised against $\alpha$-VAChT $(1: 1000$, rabbit polyclonal, Cat No. 139-103, Synaptic Systems, Göttingen, Germany; RRID: AB_10893979) diluted in blocking solution. The next morning, after washing $3 \times$ in TBS-T, the membrane was incubated with the secondary antibody for $1 \mathrm{~h}$ at RT. The membrane was then washed again in TBS-T before visualizing the signal using the ECL-plus kit (Cat No. RPN2236, GE Healthcare, UK) in the Bio-Rad Chemi-Doc ${ }^{\mathrm{Tm}}$ Imaging system. Following such washes, the membrane was stripped and then probed for $\alpha$-synaptophysin using a rabbit polyclonal antibody (1:2000, Cat No. 32594, Abcam, UK; RRID: AB_778204), dissolved in 5\% bovine serum albumin (BSA)/TBS-T, as an internal loading control. Protein densities on Western blots were analyzed and quantified with v. 1.45 s ImageJ software (http://imagej.nih.gov/ij/; provided in the public domain by the National Institutes of Health, Bethesda, MD, USA).

\section{Statistical analysis}

All data are expressed as mean \pm standard error of the mean (SEM). SPSS Statistics (v.22, IBM) software was used for statistical analysis of the data. The distribution of the data was examined using the D'Agostino-Pearson omnibus test. Mouse VAChT gene copies and VAChT protein comparisons between genotypes were analyzed using an independent Student's $t$ test. A one-way ANOVA followed by the Tukey's multiple comparison test was used to analyze VAChT mRNA levels between ChAT::Cre+ rats, ChAT::Cre- $(\mathrm{Wt})$ rats, and also Wt mice, which served as an additional control for the VAChT mRNA measures. Corticosterone, leptin and glucose data were analyzed a using mixed-effects ANOVA with time-point being the within-subjects factor and genotype the between-subjects factor. Freezing scores for fear conditioning and all anxiety profile task parameters were compared using an independent samples $t$ test. Baseline 5-CSRTT data were analyzed in a mixed-effects ANOVA with session as within-subjects factor and genotype as between-subjects factor. The varied SD probe was analyzed similarly with session and SD time as within subject and genotype as between subject factors. Any main effects were examined using the Tukey's HSD (honestly significant difference) post hoc comparison, with interactions that were explored using a Fisher's LSD (least significant difference) post hoc analysis; from both tools, the effect size $\left(\eta^{2}\right)$ was calculated. Any extreme outliers were identified using the 'outlier labeling rule' and removed from data analysis (Hoaglin et al. 1986). The rule entails that the difference between the lower and upper quartile is multiplied by a factor ' $g$ '. Due to the published advice advising that the initially suggested ' $g$ ' value of 1.5 (Hoaglin et al. 1986) proved inaccurate in some instances and that a multiplier value of 2.2 generates valid results more often (Hoaglin and Iglewicz 1987), we utilized a ' $g$ ' value of 2.2 to the datasets generated for the current project. $p$ values were designated as: $* * * p<0.001$, extremely significant; ${ }^{* *} p \leq 0.01$, highly significant; $* p \leq 0.05$, significant and $p$ $>0.05$, non-significant (NS).

\section{Results}

\section{ChAT::Cre+ rats exhibit an anxiolytic-like and hypermobile behavioral profile}

Anxiety profiles were evaluated from assessing the rats' behavior in the OF, EPM and the LDB. The behavioral time-line for anxiety profiling is shown in Fig. 1a. In the OF, ChAT::Cre+ rats displayed a hypermobility profile compared to ChAT::Cre- siblings, with a significantly increased average traveling speed $(* * * p=0.0008)$ (Fig. 1b), a greater distance covered $(* * p=0.0063$ ) (Fig. 1c), as well as rearing more often $(* * * p=0.0006)$ (Fig. 1d) and, relatedly, spending more time rearing $(* * * p=0.0006)$ (Fig. 1e). The ChAT::Cre+ also appeared anxiolytic by entering the inner diameter significantly more than ChAT: $\mathrm{Cre}-$ siblings $(* * * p$ $=0.0002$ ) (Fig. 1f), while also spending significantly more time within the inner field of the test arena, compared to the ChAT::Cre- rats $(* * * p=0.0002)$ (Fig. 1g).

Similarly, ChAT::Cre+ rats showed higher average speed of movement ( $* p=0.014$ ) (Fig. 2a) and covered more distance in the EPM than their ChAT::Cre- siblings $(* * * p=$ 0.0005) (Fig. 2b) over the entire $5 \mathrm{~min}$ that rats were tested in the EPM; these findings again suggested a general hyperlocomotion profile. In this test, a similar trend was also seen to that in the OF test, for ChAT::Cre+ rats to rear more $(* * p$ $=0.0017)($ Fig. $2 \mathrm{c})$ and for longer $(* p=0.0134)($ Fig. $2 \mathrm{~d})$ for the full testing period within the EPM, than was seen for ChAT::Cre- rats. ChAT::Cre+ rats' general anxiolytic tendency was reinforced by displaying an increased tendency to visit the open arms of the EPM more often than ChAT::Cre- rats $(* * * p=0.0006)($ Fig. $2 \mathrm{e})$ and spending significantly more time in the open arms of the maze $(* p=$ 0.0403) (Fig. 2f) compared to ChAT::Cre- rats.

In the LDB, ChAT::Cre+ rats entered the light chamber significantly more frequently $(* p=0.0132$ ) (Fig. 3a), exhibited greater average duration of time in the light chamber ( ${ }^{*} p=0.0118$ ) (Fig. $3 \mathrm{~b}$ ) and subsequently, also spent less time in the dark chamber of the test apparatus $\left({ }^{*} p=0.0277\right)$ (Fig. 3c). Taken together, use of multiple experimental paradigms revealed that the ChAT::Cre+ rat model displays spontaneous locomotion and adaptation to a novel, anxiety-evoking environment that differs from 
Fig. 1 The rats' behavior was assessed for $10 \mathrm{~min}$ in the $\mathrm{OF}$ arena to measure locomotion and anxiety-like behavior. a The timeline of the anxiety-related and locomotion test procedures performed on the rats are shown; all rats performed the same behavioral tests in the order shown. To investigate the acute effect of foot shock at post-acquisition and post-retention stage on plasma corticosterone, leptin and glucose levels, blood samples were collected at the time-points shown, to compare to baseline levels. In the OF test, the behavioral profile of ChAT::Cre+ rats $(n=8)$ differed from their ChAT::Cresiblings $(n=10)$. Taken together, the parameters $\mathbf{b}$ average speed of movement, $\mathbf{c}$ total distance traveled, $\mathbf{d}$ number of rearings made and also $\mathbf{e}$ the time spent rearing during the test, indicated that ChAT::Cre+ rats were hypermobile. Other parameters like $\mathbf{f}$ number of entries into inner field and $(\mathrm{g})$ time spent in the inner field, further revealed that ChAT::Cre+ rats were in a mixed hypermobile and anxiolytic state. All data are presented as the mean \pm SEM. $* p \leq 0.05, * * p \leq 0.01$ and $* * * p<0.001$, compared to ChAT::Cre- rats

(a)

\begin{tabular}{|c|c|c|c|c|c|c|c|c|c|}
\hline Time (days (d)) & d1 & d8 & d18 & d39 & $\mathrm{d} 46$ & $\mathrm{~d} 46+25 \mathrm{~min}$ & $\mathrm{~d} 47$ & $\mathrm{~d} 47+25 \mathrm{~min}$ & d54 \\
\hline Behavioral test & L OF & L ЕРм & L LDB & \begin{tabular}{|l} 
Blood \\
sampling \\
at baseline
\end{tabular} & $\begin{array}{l}\text { L CFCT: } \\
\text { Acquisition } \\
\text { phase }\end{array}$ & $\begin{array}{l}\text { L Blood } \\
\text { sampling }\end{array}$ & $\begin{array}{l}\text { LFCT: } \\
\text { Retention } \\
\text { phase }\end{array}$ & $\begin{array}{l}\text { Blood } \\
\text { sampling }\end{array}$ & riff \\
\hline
\end{tabular}

(b)

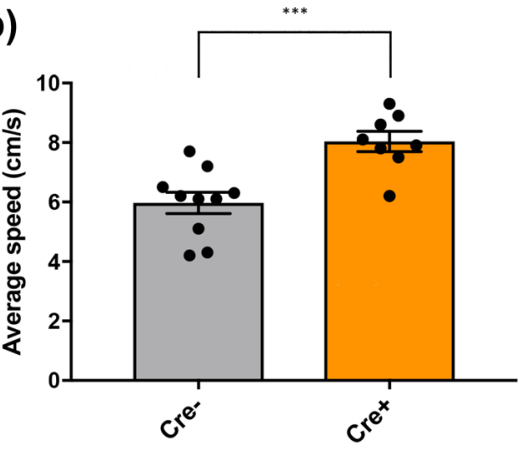

(d)

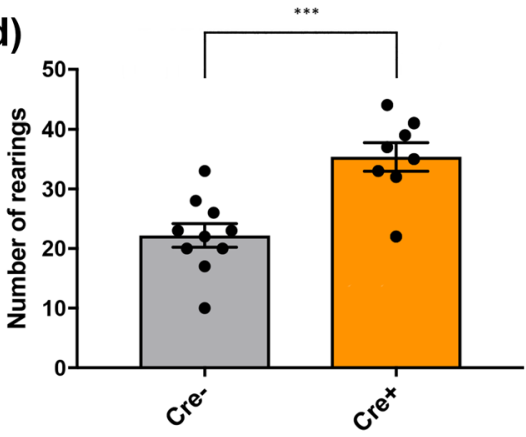

(f)

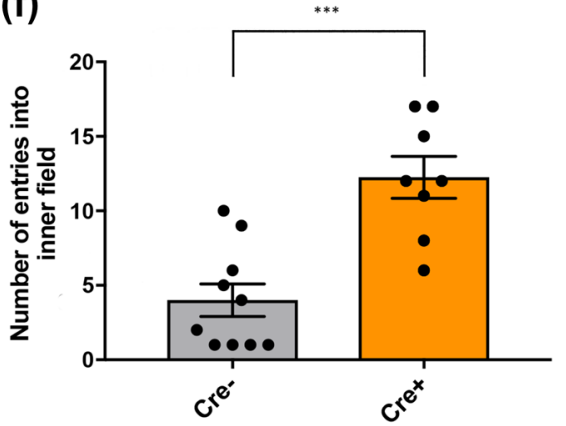

(c)

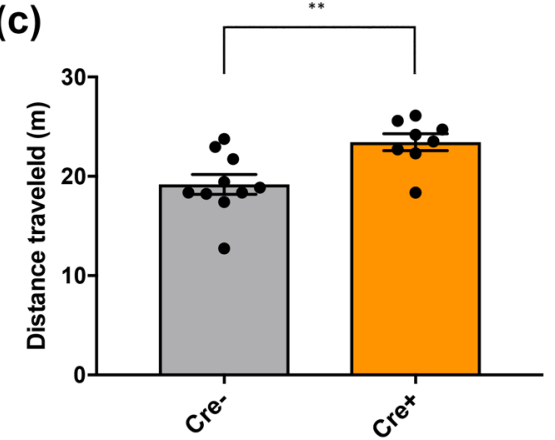

(e)

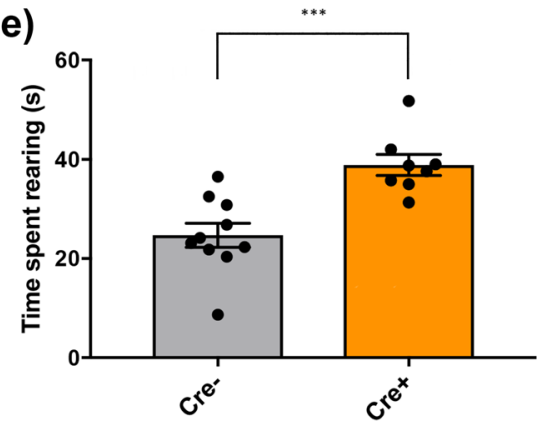

(g)

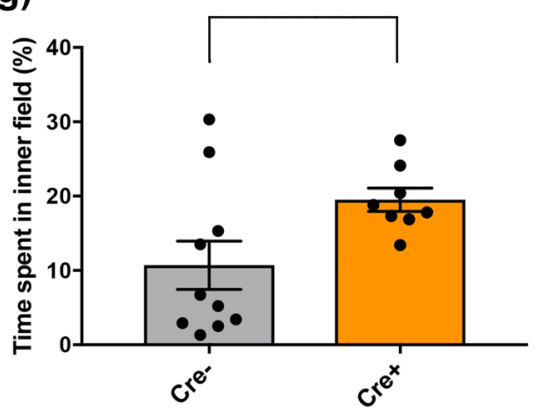

the baseline behavioral profile seen for non-transgenic littermate rats of a similar strain.

None of the rats' performance scores were excluded from the final analysis for the anxiety-profiling tasks, based on the exclusion criteria set for each behavioral measure (for a description of these, please see the "Materials and methods"). In addition, no animal died during behavioral testing. Hence, data collected for all rats tested in the OF, EPM and LDB were included in the final analyses.

ChAT::Cre+ rats make more errors of omission and fewer perseverative responses than Chat::Cre- rats in the 5-CSRTT.
Figure 4 summarizes the results of the behavior displayed by the two genotypes in the 5-CSRTT. No differences were detected between the two groups in terms of choice accuracy ( $p=0.714$, NS) (Fig. 4a), the number of correct responses ( $p=0.105$, NS) (Fig. $4 \mathrm{~b})$ or the number of incorrect responses ( $p=0.814$, NS) (Fig. 4c). Relatedly, the measures 'mean delay to make a correct response' ( $p$ $=0.364, \mathrm{NS})($ Fig. $4 \mathrm{~d})$ or to make an incorrect response ( $p$ $=0.061$, NS) $($ Fig. 4e) were also left unchanged between transgenic and non-transgenic rats. Furthermore, the measures 'number of premature responses' ( $p=0.946, \mathrm{NS}$ ) (Fig. 4f) and 'mean latency of reward collection following a correct response' $(p=0.308$, NS) (Fig. $4 \mathrm{~g})$ were also 
Fig. 2 Anxiety-like behavior in the rats was further assessed in the EPM maze, where the task is based on an approachavoidance conflict and greater avoidance of the open arms is interpreted as an exhibition of higher anxiety levels. The findings reflected those obtained for OF testing, with regards to both general ambulation and anxietylike behavior. Over the duration of the test (5 min), ChAT::Cre+ rats $(n=8)$ traveled on average at $\mathbf{a}$ greater speed and $\mathbf{b}$ covered a greater distance than the ChAT::Cre- rats $(n=10)$. Compared to ChAT::Cre- rats, ChAT::Cre+ ones also revealed hypermobility by making c significantly more rearing bouts and $\mathbf{d}$ spending more time rearing. ChAT::Cre+ rats also made e more entries into the open arms of the EPM apparatus and f spent more time in the open arms than their ChAT::Cresiblings, reflecting the general anxiolytic profile detected for the ChAT::Cre+ rats seen during testing in the OF. All data are presented as the mean \pm SEM. $* p \leq 0.05, * * p \leq 0.01$ and $* * * p<0.001$, compared to ChAT::Cre- rats (a)

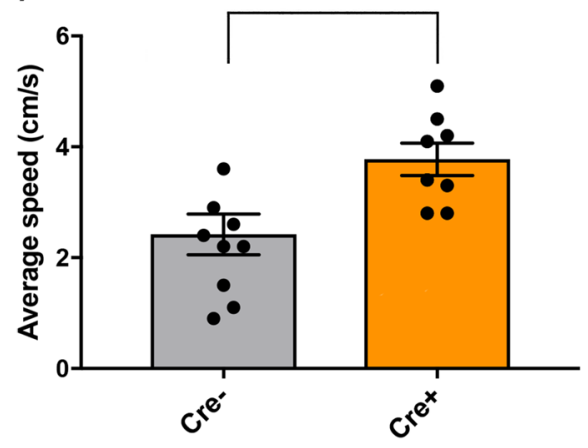

(c)

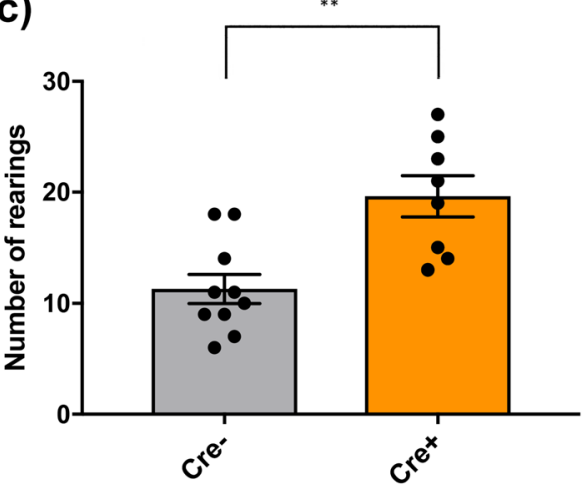

(e)

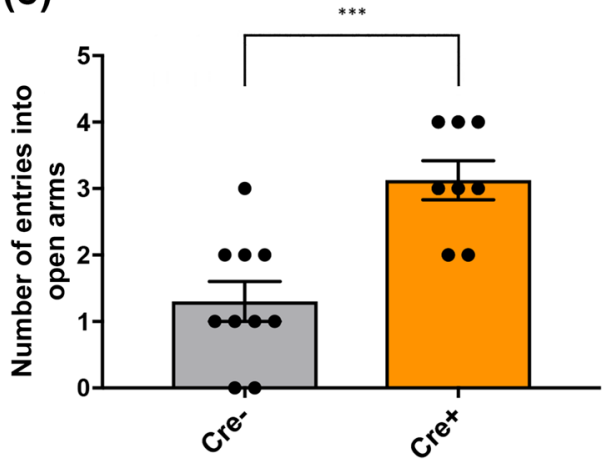

(b)

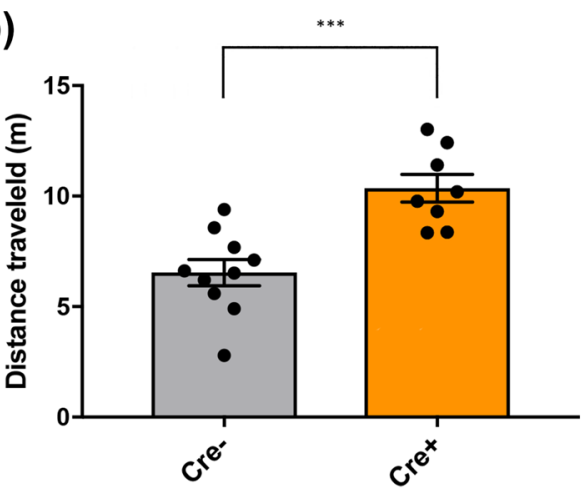

(d)

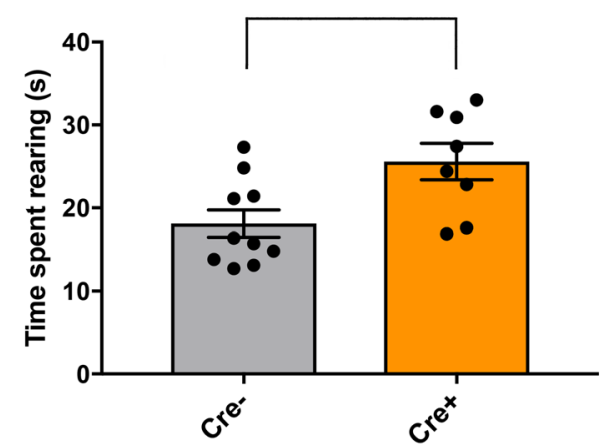

(f)

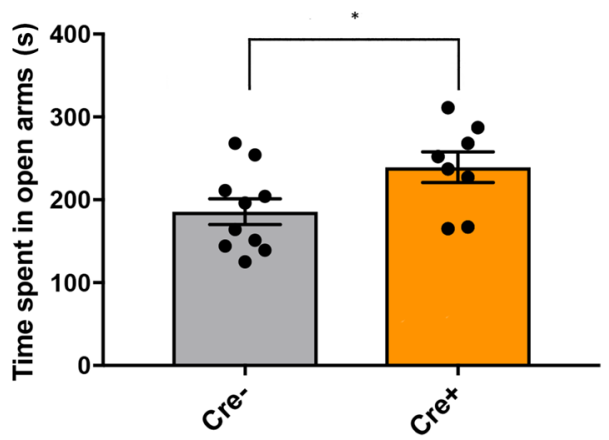

similar between ChAT::Cre+ and ChAT::Cre- rats. However, compared to their ChAT::Cre- siblings, ChAT::Cre+ rats made significantly more errors of omission $\left({ }^{*} p=0.016\right.$, $\eta^{2}=0.373$ ) (Fig. 4h), but fewer perseverative responses ( $* * p$ $=0.006, \eta^{2}=0.456$ ) (Fig. 4i).

During a version of the 5-CSRTT, where the SD probe length was varied, a main effect of SD on accuracy was detected $(* * p=0.002)$ (Fig. 5a) that featured as decreasing accuracy as SD times became shorter. This effect was caused by the overall tendency of ChAT::Cre+ rats to make fewer correct responses ( $* p=0.05$ ) (Fig. 5b), implying more incorrect responses $(* * p=0.009)$ (Fig. 5c) as the SD decreased, compared to ChAT::Cre- siblings. However, no significant interaction was detected between SD variation and genotype. Between-subject genotype analysis showed that upon variable and, therefore, increased demand conditions, ChAT::Cre+ rats made fewer perseverative responses compared to ChAT::Cre- ones $\left({ }^{*} p=0.018, \eta^{2}=0.410\right)$ (Fig. 5d). This was similar to baseline behavior, to indicate that this phenotypical difference could be regarded as robust. When comparing perseverative responses assessed for different SD times, this decrease was significant at the longest duration probe tested for, namely $0.15 \mathrm{~s}(* * p=0.005)$ (Fig. $5 \mathrm{~d})$. The tendency for ChAT::Cre+ rats to omit more responses compared to their baseline behavior was not seen in the varied SD probe ( $p=0.287, \mathrm{NS}$ ) (Fig. 5e). Differences were also not seen between genotypes in the varied SD probe when 
Fig. 3 The LDB is another conventional anxiety test that is based on the innate aversion of rodents to brightly illuminated areas, which was utilized to test unconditional anxiety responses in the rats. The ChAT::Cre+ rats $(n=8)$ showed a greater preference for the light compartment of the box compared to their ChAT::Cre- siblings ( $n$ $=10$ ). Behavioral parameters assessed were: a total number of entries made into the light chamber, $\mathbf{b}$ time spent in the light chamber and c latency time for rats to enter the light chamber. All data are presented as the mean \pm SEM. $* p \leq 0.05$, compared to ChAT::Cre- rats
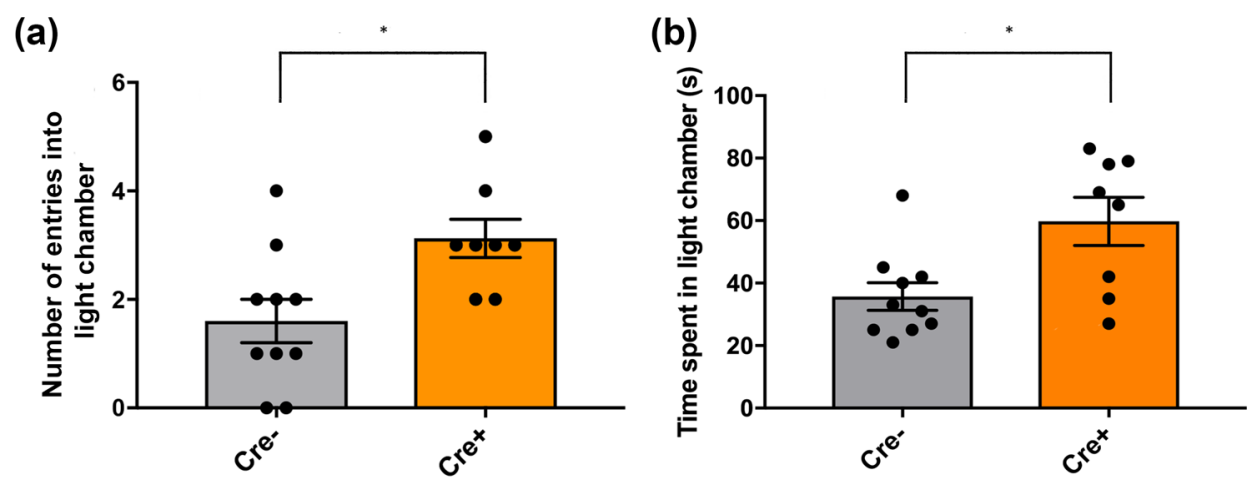

(c)

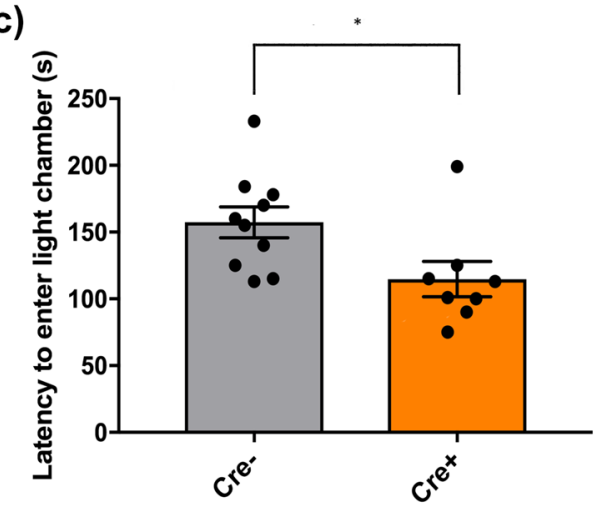

number of premature responses were assessed ( $p=0.389$, NS) (Fig. 5f).

\section{ChAT::Cre+ rats display a biologically intact stress response to an acute stressor in a CFCT paradigm}

Previous studies showed that the cholinergic system could play a role in HPA activation and corticosterone levels (Newman et al. 2001; Paul et al. 2015). To investigate the effects of altered cholinergic modulation on physiological stress responses, we measured plasma levels of corticosterone at baseline and post-retention testing in the CFCT. With this regard, several studies have demonstrated a positive relationship between circulating corticosterone levels and contextual conditioning (Pugh et al. 1997; Thompson et al. 2004).

The level of freezing, measured during retention testing, did not show any significant difference between genotypes ( $p=0.642, \mathrm{NS})$ and produced an average of $\sim 90 \%$ for each group (Fig. 6a). When all rats were considered together, without accounting for genotype, a significant increase of corticosterone from baseline to fear acquisition was seen $\left({ }^{*} p<0.05\right)$, and also when baseline levels were compared to levels reached at the retention phase of the CFCT $(* * * p$ $<0.001$ ) (Fig. 6b). However, this effect was independent of genotype $(p=0.618, \mathrm{NS})$, with no interaction seen between genotype $\times$ sample point either $(p=0.199$, NS $)$. Post hoc analysis revealed high statistical significance between differential levels of circulating corticosterone levels from baseline to retention phase for ChAT::Cre- litter mates $(* * * p<0.001)$; however, a similar comparison made for ChAT::Cre+ rats proved non-significant $(p=0.057, \mathrm{NS})$ (Fig. 6b).

Analysis of plasma glucose levels revealed no significant difference between sample points ( $p=0.196$, NS), when comparing the genotypes ( $p=0.095, \mathrm{NS})$ or at a sample point $\times$ genotype interaction level $(p=0.290$, NS) (Fig. 6c). Analysis of leptin levels revealed no significant difference in main effect of sample point ( $p=0.50, \mathrm{NS})$ or of genotype $(p=0.933$, NS), while no significant interaction effect was observed either ( $p=0.108$, NS) (Fig. 6d).

\section{Higher levels of VAChT mRNA and protein expression in ChAT::Cre+ rats compared to Wt rats}

qPCR results revealed that all Long-Evans ChAT::Cre+ rats analyzed here contained two copies of the mouse VAChT gene relative to ChAT::Cre- rats $(* * * p<0.001)$ (Fig. 7a). Mouse VAChT mRNA was variable within individual rats, with some having levels similar to mouse controls (Fig. 7b). However, overall, the expression of mouse VAChT mRNA in ChAT::Cre+ rats achieved approximately $50 \%$ the levels of mouse controls (Fig. 7b), which indicates the expression of the mouse VAChT gene inserts in the rat genome. Supporting 
Fig. 4 Performance in the 5-CSRTT assessed for differences in attention, impulsivity and cognitive flexibility between the transgenic and Wt rats. ChAT::Cre+ rats ( $n$ =13) made significantly more omission errors and fewer perseverative responses than their ChAT::Cre - siblings ( $n$ $=8$ ) during 5-CSRTT testing, which measured the following parameters: a choice accuracy; b average number of correct responses; $\mathbf{c}$ average number of incorrect responses; $\mathbf{d}$ latency to make a correct nose poke response following stimulus presentation; e latency to make an incorrect response following stimulus presentation; $\mathbf{f}$ average number of premature responses; g latency to collect sugar pellet reward from food magazine after a correct response; $\mathbf{h}$ average number of errors of omission and $\mathbf{i}$ average total number of perseverative responses. All data are presented as the mean \pm SEM. ${ }^{*} p \leq 0.05,{ }^{*} * p \leq 0.01$, compared to ChAT::Cre- rats (a)

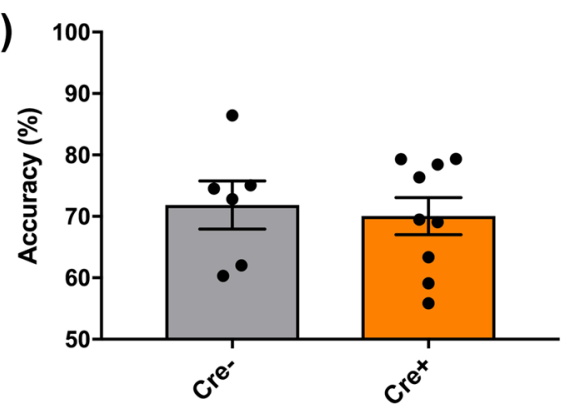

(c)

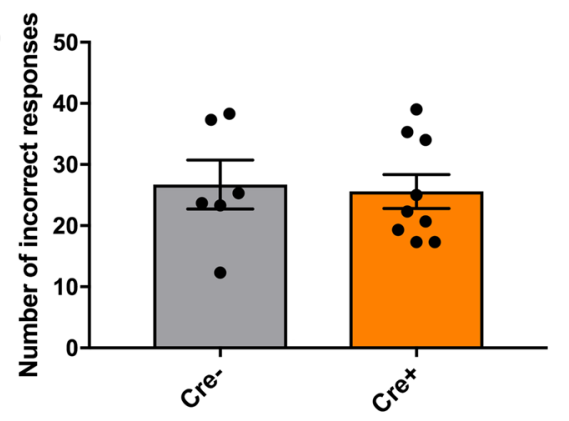

(e)

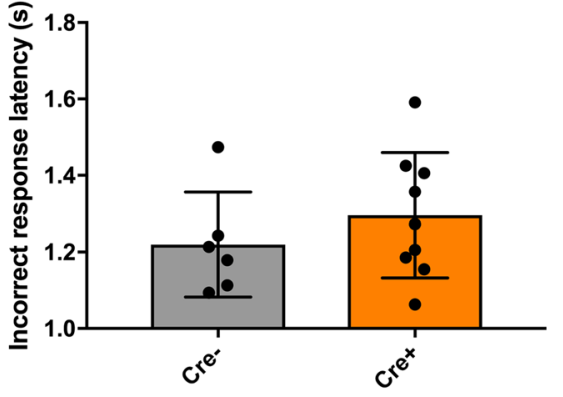

(g)

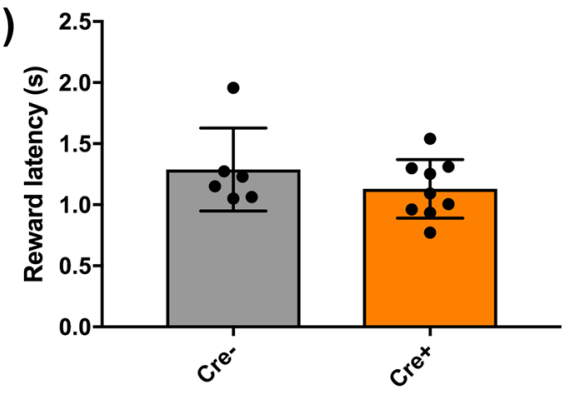

(b)

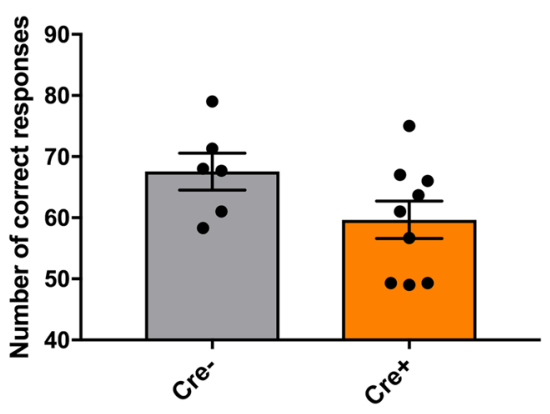

(d)

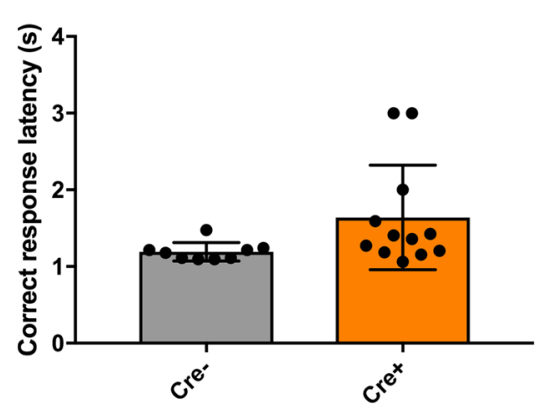

(f)

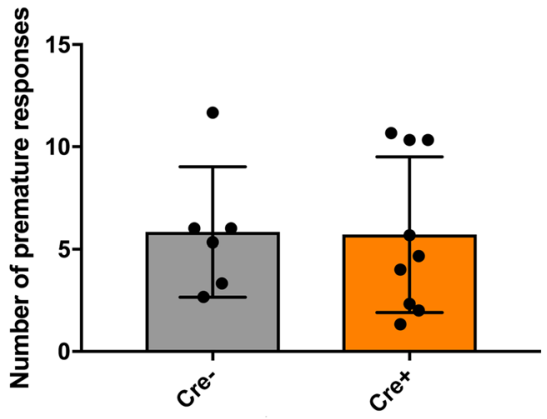

(h)

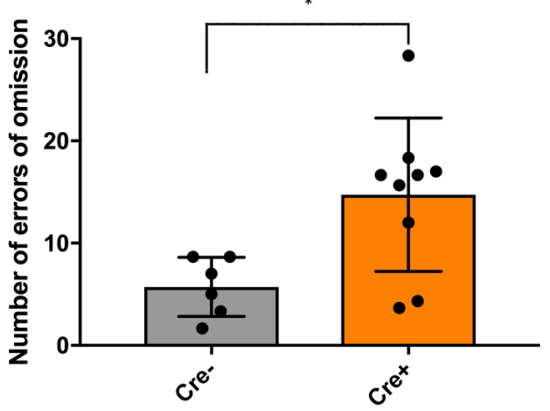

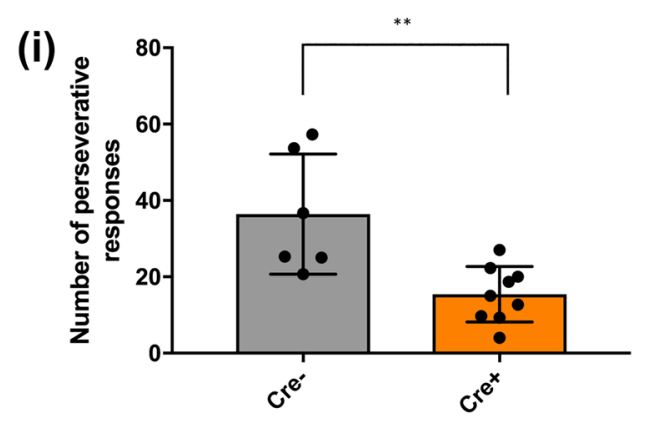


Fig. 5 A version of the 5-CSRTT, where SD length was varied (0.6, 0.3 and $0.15 \mathrm{~s})$, further measured attentiveness, impulsivity, processing speed and distractibility in the rats. SD length variance exerted an overall significant effect on a choice accuracy. This was since, compared to ChAT::Cre- rats $(n=8)$, the ChAT::Cre+ ones $(n=13)$ made on average, $\mathbf{b}$ less correct responses and, therefore, $\mathbf{c}$ more incorrect responses, as SD decreased. d ChAT::Cre+ rats made less perseverative responses compared to ChAT::Cre- ones, with the number of perseverative responses that decreased most significantly at the longest duration probe, namely $0.15 \mathrm{~s}$. e As detected in the standard 5-CSRTT, a tendency by ChAT::Cre+ rats to omit more responses compared to baseline behavior was not repeated. f Groups revealed similar levels of premature responses. All data are presented as the mean \pm SEM. $* * p \leq 0.01$, compared to ChAT::Cre- rats (a)

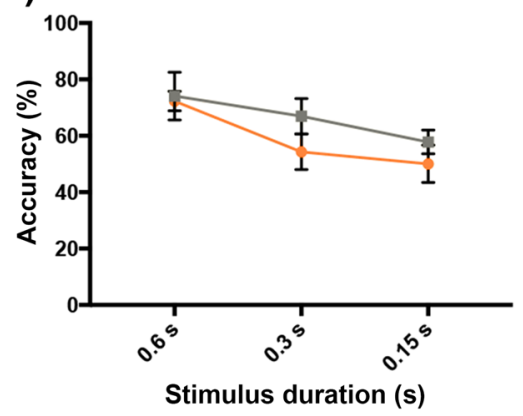

(c)

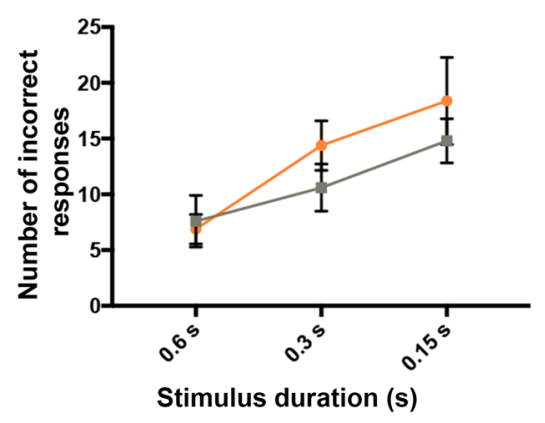

(e)

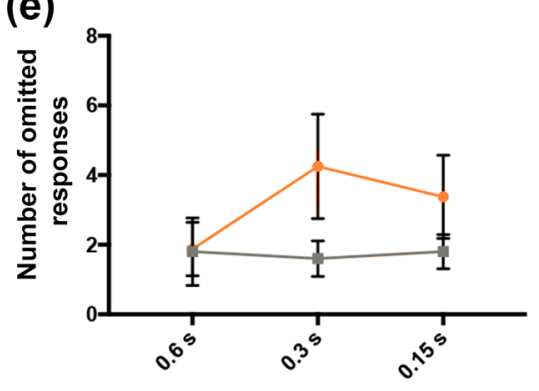

Stimulus duration (s) (b)

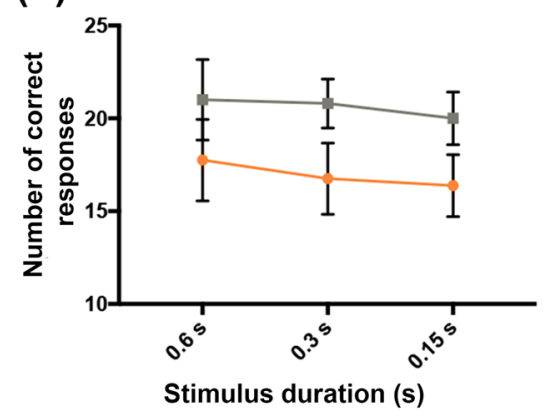

(d)

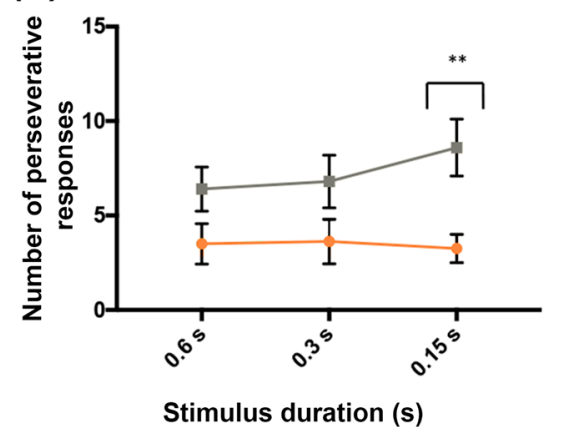

(f)

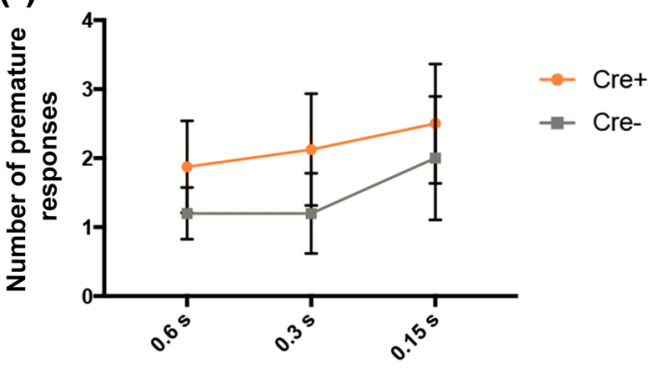

Stimulus duration (s) the gene copy and the mRNA analysis, VAChT protein levels were increased close to $50 \%$ in ChAT::Cre+ rats when compared to Cre- siblings $(* * p=0.004)$ (Fig. 7c). This result supports the notion that the increased VAChT expression found in ChAT::Cre+ rats may also increase ACh levels within synaptic vesicles, as had previously been reported for BAC transgenic mice that express channelrhodopsin protein, a light-gated ion channel, under control of the ChaT promoter (Sugita et al. 2016).

\section{Discussion}

The current findings show that, compared to their Wt siblings, Long-Evans ChAT::Cre+ rats exhibit altered baseline behavior, affecting gross locomotor function, anxiety-related behavior as well as some aspects of sustained attention. We also molecularly analyzed the PFCs collected from the rats, with this brain region that receives dense cholinergic innervation and also connects directly and indirectly via cholinergic projection neurons with multiple brain areas, to provide important modulation of cortical function (Bloem et al. 2014; Tingley et al. 2015). The analysis revealed that these behavioral changes were paralleled by altered expression of VAChT within the PFC, at both a gene and protein level, with VAChT being a key molecular mechanism in the transport machinery of ACh from the cytoplasm to the lumen of synaptic vesicles, to ultimately release ACh into the synaptic cleft. Physiologically, an increase in levels of this functional transporter could alter ACh levels at the synapse (Kolisnyk et al. 2013; Sugita et al. 2016; Nagy and Aubert 2012), which could alter cholinergic tone and, subsequently, behavioral phenotype in rodents. Hence, the current findings demonstrate that expression of the ChAT-BAC construct makes these rats different from their Wt siblings, similar to findings previously reported using BAC transgenic 
(a)

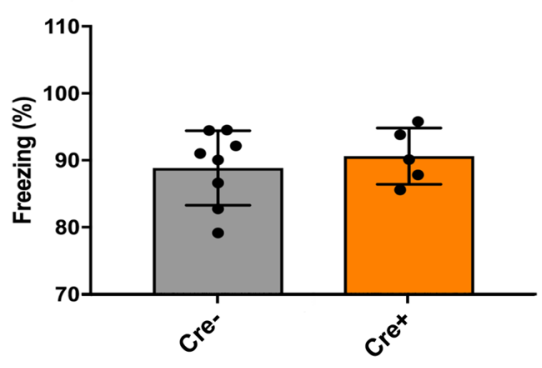

(c)

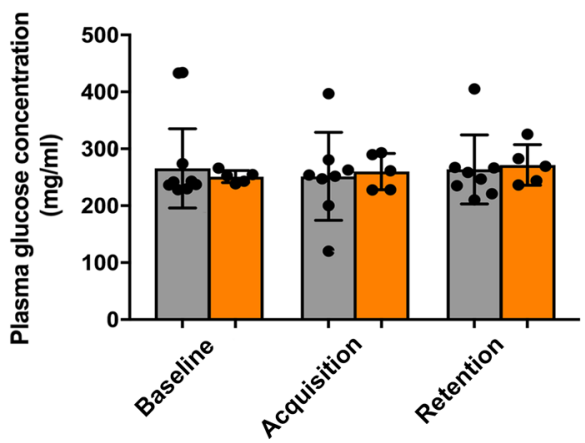

Fig. 6 Freezing behavior and blood plasma analysis of HPA-axis biomarkers performed on ChAT::Cre+ $(n=5)$ and ChAT::Cre- $(n=8)$ rats at baseline ( 2 days before initiating the task protocol), with testing that was then repeated at the fear acquisition and fear retention stages of a CFCT. a Freezing scores acquired for retention testing; b comparison of corticosterone blood plasma levels between genotypes at the different CFCT testing phases; $\mathbf{c}$ a similar comparison made for glucose blood plasma levels and for $\mathbf{d}$ leptin blood plasma

mice engineered to express a light-sensitive protein under control of the ChAT promoter (Kolisnyk et al. 2013; Chen et al. 2018).

As was reviewed by Prado et al. (2013), ACh synthesis depends on the uptake of the ACh precursor, choline by CHT1 [high-affinity choline transporter/SLC5A7 (solute carrier family 5 member 7)] that mainly expresses within cholinergic neurons, yet presents in certain non-neuronal cells also. In the cytoplasm of synapses, ACh is then synthesized by the enzyme ChAT, with ACh that is then loaded into synaptic vesicles by VAChT. Upon arrival of a nerve impulse, the vesicles fuse to the plasma membrane and release ACh that signal through nicotinic and muscarinic cholinergic receptors. ACh is rapidly degraded into acetate and choline by the enzyme AChE (acetylcholinesterase). In the current work, we have only evaluated expression levels of VAChT, in line with other studies utilizing BAC transgenic mice for expressing a light-sensitive (b)

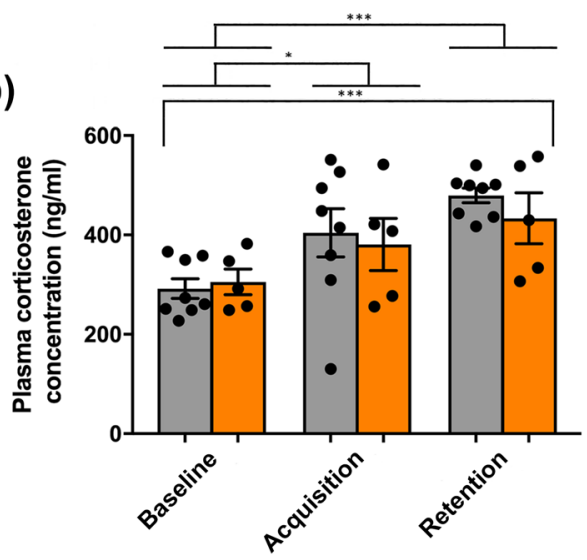

(d)

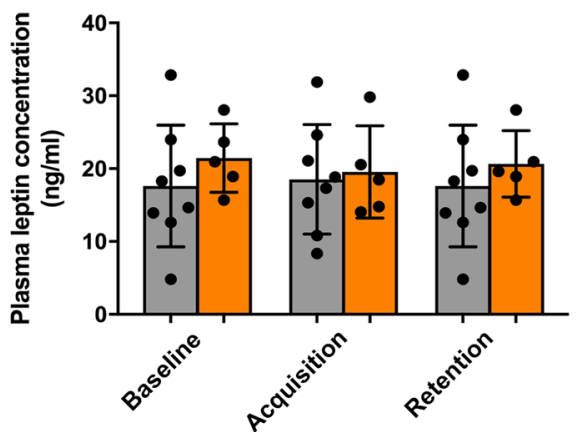

levels. The significant increase in blood plasma corticosterone levels seen in both rat genotypes from baseline to a repeat analysis soon after rats completed the fear acquisition and also the fear retention test phases, indicates that all rats of this strain, regardless of the genotype (ChAT::Cre+ vs. Wt), respond in a biologically intact manner to aversive conditioning. All data are presented as the mean \pm SEM. $* p$ $\leq 0.05$ and $* * * p<0.001$, compared to both genotype rats' baseline measurements

protein under control of the ChAT promoter that also restricted molecular analysis to VAChT, as a valid indicator of cholinergic tone (Kolisnyk et al. 2013; Chen et al. 2018). The importance of functional vesicular storage and synaptic "loading" of ACh, which is modulated by VAChT, acts as a required step for ACh release. In addition, decreased VAChT expression has been shown to lead to several motor and cognitive dysfunctions (Prado et al. 2006; Martyn et al. 2012), some of which was presently behaviorally evaluated for in the ChAT::Cre+.

To ensure a comprehensive understanding of the levels at which altered cholinergic metabolism and transmission might be altered as a result of the ChAT::Cre+ genotype, future work should undertake gene and protein expression analysis of other important mediators in this process, including but not limited to ChAT, CHT-1, AChE, but also muscarinic and nicotinic receptors for signaling ACh. Such eventual comprehensive profiling will inform greatly on the 
Fig. 7 Measurement of prefrontal cortical VAChT mRNA and protein levels. a qPCR results indicated additional mouse VAChT gene copies in all ChAT::Cre+ rats $(n=5)$ compared to ChAT::Cre- controls $(n=8)$. This translated to $\mathbf{b}$ increased mouse VAChT mRNA expression in $\mathrm{Cre}+(n$ $=5)$ and Cre- $(n=7)$ siblings compared to Wt mice $(n=4)$ and also $\mathbf{c}$ increased VAChT protein expression in PFC brain samples of Cre+ $(n=5)$ and Cre- $(n=8)$ rats. All data are presented as the mean \pm SEM. $* p \leq 0.05, * * p \leq 0.01$ and $* * * p<0.001$, compared to ChAT::Cre - rats and Wt mouse
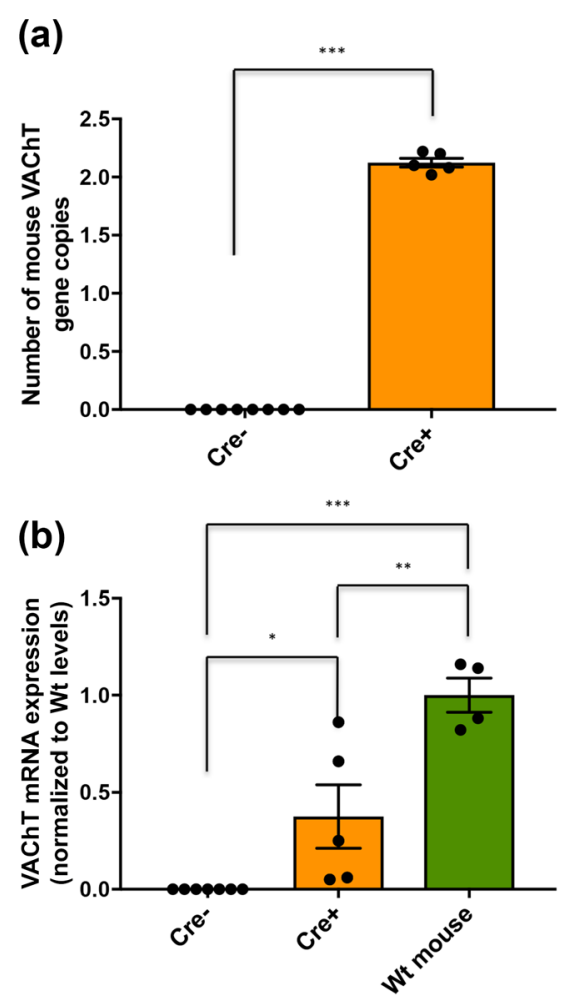

(c)

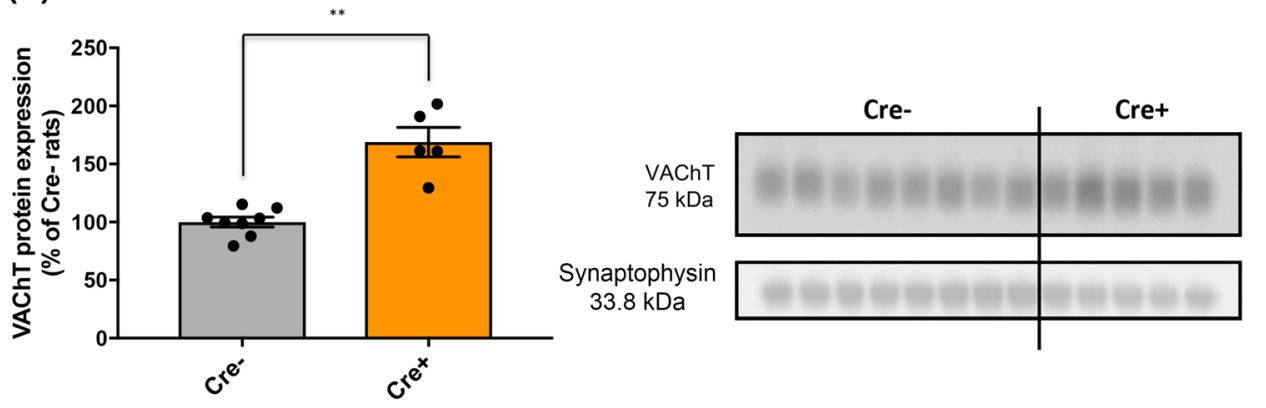

design of experimental work involving this popular experimental rat tool.

Our data indicated a hypermobile state for ChAT::Cre+ rats over Wt ones in several parameters assessed in the OF, EPM and LDB. The observed locomotor arousal in the transgenic rats is consistent with the previously proposed mechanism that instantaneous ACh synaptic release correlates positively with increased activity by rodents seen in novel environments (Mizuno et al. 1991; Cohen et al. 2012), while ACh is known to fulfill a complex role in locomotor control, which includes modulating the dopaminergic system (Rice and Cragg 2004). Confirmation of the specific mechanisms for the observed hyperactive spontaneous activity awaits brain region-specific VAChT overexpressing rat models.

This result differs from those presented by Azzopardi et al. (2018) who observed no hyperlocomotive phenotype in ChAT::Cre+ rats, although enhanced motor endurance had previously been reported for the ChAT-ChR2-eYFP (enhanced Yellow Fluorescent Protein) BAC transgenic mouse line (Kolisnyk et al. 2013). It is worth noting though that the study by Azzopardi et al. (2018) made use of a single metric (distance traveled) for assessing abnormal mobility, with the group using a $45 \times 45 \mathrm{~cm}$ square OF box. Instead, we made use of a circular test apparatus, with the shape of OF arena (circular, square or rectangular) that has been identified as an important variable that can influence OF performance by rodents (Sestakova et al. 2013). Similarly, this research group only assessed the time spent in the outer perimeter in the same apparatus as indicator of anxiety. Lastly, Azzopardi et al. (2018) utilized rats in the experimental procedures at a slightly older age, at 10-14 weeks, whereas rats used in the current study ranged between 8 and 10 weeks of age. However, Long-Evans rats are considered to have reached adulthood by 8 weeks of age (Snyder et al. 2009; Jackson et al. 2016), while the age ranges of rats used in the present work do overlap somewhat with 
that of the study by Azzopardi et al. (2018), allowing for reasonable confidence that the differences in experimental outcomes between these two studies is likely and at least not exclusively due to age-related differences. However, differences in the age of experimental rats have been highlighted as a potentially critical experimental variable, to especially account for changes in neurotransmitter release and receptor activation, that appear to alter substantially, especially over early stages of development (McCutcheon and Marinelli 2009). Considering the differences in testing conditions and the characteristics of the experimental rats used in the current study compared to those used by Azzopardi et al. (2018), we conclude that our ambulation and anxiety results may not be directly comparable.

Our results also reveal ChAT::Cre+ rats to be anxiolytic, as was assessed in three frequently used tests of anxiety-like behavior, with such tests that consistently demonstrate the ability to reveal anxiolytic or anxiogenic profiles of rodents using either pharmacological- or transgenic manipulations (Crawley 1985; Belzung and Griebel 2001; Prut and Belzung 2003). This result suggests that modest levels of $V A C h T$ overexpression at both the gene and protein level, previously shown to increase $\mathrm{ACh}$ release and, therefore, render animals hypercholinergic (Sugita et al. 2016; Janickova et al. 2017), associate with an anxiolytic behavioral profile in the ChAT::Cre+ rats. The findings reflect those from another hypercholinergic mouse model that also overexpresses VAChT at the gene and protein level, with such mice that showed enhanced exploration of novel environments and, therefore, anxiolytic-like behavior, when compared to C57BL/6J (B6) control mice (Nagy and Aubert 2013). In this work, use was made of the B6eGFPChAT congenic mouse model that contains four genomic copies of the cholinergic gene locus, which encompasses the promoter and coding regions of both VAChT and ChAT (Tallini et al. 2006; Nagy and Aubert 2012). As earlier work demonstrated increased VAChT gene and protein expression in congenic B6eGFPChAT mice (Nagy and Aubert 2012), this experimental model allows for evaluating whether increasing the vesicular storage and release of ACh is sufficient for eliciting changes in behavioral activity.

Relatedly, previous studies showed that exposure to novel stimuli (i.e., novel environments) associates with cholinergic activation and increased ACh release (Thiel et al. 1998; Giovannini et al. 2001), while others showed that modulation of central cholinergic systems regulate emotional processes including anxiety and stress (Podhorna and Franklin 1999, 2000; Janickova et al. 2019). However, the effects of ACh on anxiety-like behavior in rodents has proved complex, with increased ACh release that evoked both anxiolytic and anxiogenic reactions (File et al. 1998, 2000), which may relate to brain region-specific configurations of ACh receptors (File et al. 2000; Labarca et al. 2001).
The cholinergic system has been shown to play an important role in attentional mechanisms. Selective lesioning of the Ch5 cholinergic cell group of the pedunculopontine nucleus (PPN) (Mesulam et al. 1984), a rostral brainstem nucleus, resulted in a deficit shown during tests assessing for sustained attention, which manifested as a significant decrease in the number of correct responses and an increase in response latency (Cyr et al. 2015). Similarly, cholinergic-specific lesions made in rats to the $\mathrm{Ch} 4$ cholinergic cell group located in the nucleus basalis magnocellularis (NBMc), the rat homologue of the nucleus basalis of Meynert (NBM), by means of 192 IgG-saporin infusions, also produced attentional impairments along with decreased levels of ACh in the PFC (McGaughy et al. 2002). Moreover, attentional impairments are deemed a core feature of AD and have been shown to be caused, at least in part, by damage sustained by the NBM, which undergoes significant neuronal loss during progressive $\mathrm{AD}$ (Ferreira-Vieira et al. 2016).

Kolisnyk et al. (2013) showed that BAC transgenic mice generated to express the light-gated ion channel, channelrhodopsin-2, under control of the ChAT promoter, with such animals that are frequently used for dissecting out the relative contribution of cholinergic circuits in behavior, display severe deficits in several domains of cognitive function, and that this may be due to VAChT overexpression seen in such mice. In particular, transgenic mice were impaired in tasks assessing the domains spatial and working memory. Interestingly, Nagy and Aubert (2015) reported, using hypercholinergic congenic B6eGFPChAT aged mice, improved spatial memory acquisition during testing in the Morris water maze, to reveal a consensus between VAChT overexpression and improved spatial navigation during normal aging. The study further demonstrated an association between the mice's augmented memory acquisition and enhanced dendritic ramification of adult-born neurons seen in the hippocampus, and at progressive ages. Since cholinergic signaling was previously shown to contribute to the dendritic expansion of target neurons (Höhmann et al. 1991), the study by Nagy and Aubert (2015) supports a role for cholinergic input to the hippocampus as a mechanism underlying the formation of dendritic networks to support formation of spatial memories.

Kolisnyk et al. (2013) also reported that the transgenic mice exhibited significantly more premature responses during the 5-CSRTT, a behavioral paradigm frequently used for evaluating attention and impulsivity in animal models. This result differed from our current findings, which did not detect a similar deficit for the transgenic rats when subjected to either the self-paced version of the 5-CSRTT, or when the SD was varied during testing. Interestingly, increased premature responding has been associated with depletion of another monoamine neurotransmitter, serotonin (Fletcher et al. 2013; Humpston et al. 2013); hence, our 
results seemingly agree that a cholinergic imbalance in the brain do not manifest as this 5-CSRTT parameter.

However, the construct 'impulsivity' encompasses various dimensions, namely motor impulsivity which refers to acting without thinking, cognitive impulsivity referring to rapid decision taking and non-planning impulsivity, referring to a present orientation (Patton et al. 1995). Furthermore, 5-CSRTT performance differences have been reported to exist between mice and rats, including a strategy bias with rats that showed greater reliance on a temporal strategy than mice did, resulting in more guessing if their timing was poor, and manifesting as premature responses (Fletcher et al. 2007). First, for validating whether differences exist between mice and rats in 5-CSRTT performance, the protocol should be standardized, including intertrial interval (ITI) lengths. Species differences in baseline neurotransmitter concentrations could also play a role (Fitzgerald 2009). In addition, given the complexity of mechanisms underlying 5-CSRTT parameters, including impulsivity, it is unlikely that a single brain function abnormality would underlie species differences, calling for considering interacting neurochemistry systems as the plausible basis of the observed interspecies differences in 5-CSRTT performance.

Our results also show that ChAT::Cre+ rats make significantly more errors of omission than their Wt siblings, although this effect did not persist when rats were tested with a variable attentional load as a result of shorter or longer it is. Although accuracy was unaffected between the transgenic and Wt rats, this arguably points at a deficit in sustained attention, since the ChAT::Cre+ rats showed an increase in omissions, while latency parameters were left largely unaffected. Multiple studies have suggested that cholinergic signaling, especially when mediated by $\alpha 4 \beta 2$ nicotinic acetylcholine receptors, underlies altered responses in this attentional parameter measured in the 5-CSRTT (Shoaib and Bizarro 2005; Semenova et al. 2007). However, again multiple interacting neuromodulatory systems might form the neurochemical substrate of this deficit, as several studies suggested that enhanced midbrain dopaminergic activity in rats influenced trial omissions, although the direction of the effect was unpredictable (Granon et al. 2000; Agnoli et al. 2013; Boekhoudt et al. 2017). It has been suggested that the monoaminergic and cholinergic systems may play separable roles in different aspects of performance controlled by the 5-CSRTT (Robbins 2002). In the case of response omissions, motivational factors have been deemed important for driving this behavioral change, and not merely attentional deficits, with dopamine that plays an established role in reward-motivation behavior mechanisms (Salamone and Correa 2012).

We also found that ChAT::Cre+ rats made significantly fewer perseverative responses in the 5-CSRTT, with this parameter that is commonly utilized as a reference for compulsive behavior as well as cognitive inflexibility, defined as the inability to adapt to goal-directed behavior in response to changing environmental demands (Gilbert and Burgess 2008; Eagle and Baunez 2010). Deficits in the ability to flexibly update behavior are observed in various neurological and psychiatric conditions including PD (Cools et al. 2001), obsessive-compulsive disorder (OCD) (Chamberlain et al. 2008), drug addiction (Everitt et al. 2007), attention deficit/hyperactive disorder (ADHD) (Rubia et al. 2009) and pathological gambling (Koehler et al. 2013). The balance of evidence suggests that the PFC and striatum, which form part of the portico-basal-thalamic circuit, co-modulate cognitive flexibility, while a multitude of studies demonstrated that ACh transmission facilitates this process (Prado et al. 2017). With this regard, several studies have shown that cortical cholinergic manipulations selectively affect aspects of this cognitive domain with PFC nicotinic receptors that appear to play an important modulatory role in this process (Allison and Shoaib 2013). For example, in old rats (Tait et al. 2013; Nikiforuk et al. 2015), rat models of schizophrenia (Alexander et al. 2013), and in rats that sustained immunolesioning of the basal forebrain cholinergic neurons (Cutuli et al. 2009), systemic administration of cholinesterase inhibitors for augmenting cholinergic transmission, improved both reversal learning (learning an association exists between one stimulus (of a pair) and reward, to then learn a reverse association where a previously unrewarded stimulus is now rewarded) and attentional set-shifting (shifting from one mental set to another) impairments. Additional experimental evidence emphasizes a role by prefrontal $\mathrm{ACh}$ in cognitive flexibility, in particular the critical role by PFCmediated cholinergic signaling in the initial inhibition of a previously learned strategy. Ridley et al. (1994) transplanted neocortices of marmosets with cholinergic-rich neural tissue some weeks after inducing bilateral excitotoxic lesions to the NBM, where animals showed intact reversal learning during a visual discrimination task. In addition, others showed no impaired acquisition in an operant discrimination task, but deficits in reversal learning, in rats that had received selective cholinergic lesioning of the NBMc (Cabrera et al. 2006). However, the role of cholinergic signaling and the synergistic interaction between this neurochemical system and the PFC in mediating cognitive flexibility remains controversial, with some authors reporting that ablation of basal forebrain cholinergic neurons does not impair reversal learning (McGaughy et al. 2008; Tait and Brown 2008). Additional participation in cognitive flexibility by ACh within other brain areas such as the hippocampus, basolateral amygdala and posterior parietal cortex also participate in cognitive flexibility (Prado et al. 2017) may explain these controversies; however, the modulating role played by PFC ACh in this process is strengthened by a large number of studies showing that cholinergic depletion of different prefrontal 
areas results in dissociable deficits in separate forms of cognitive flexibility. Optogenetic and chemogenetic tools to modulate neuronal activity levels promise to characterize the cholinergic circuits involved this and other cognitive domains and unravel the exact role of the PFC in the process. Genetically modified rodents, including ChAT::Cre+ rats as we assessed in the current study, compliment such cell- and circuit-modifying tools, by allowing cholinergic machinery to be spatially and temporally targeted. However, as our present study demonstrates, behavioral results obtained from use of such animals should consider whether baseline behavioral changes are due to a hypercholinergic phenotype.

Cholinergic tone has also been associated with several memory- and learning-related functions. In addition, cholinergic neurons of the PFC, which we dissected out in the rats for gene and protein analysis of VAChT, have been implicated as a modulator (via cholinergic signaling) of specific aspects of memory (Croxson et al. 2011). Hence, an important direction for future research is to characterize the extent and nature of memory-related impairments in this transgenic rat line.

Although the current work did not incorporate an extensive evaluation of memory-related functions in the rats, a CFCT was included, where animals learn that certain environmental stimuli predict aversive events. This classical, Pavlovian (respondent) conditioning model provides an interface between memory and emotion (LeDoux 2000). In this testing paradigm, we measured the frequency of a defensive reflex namely freezing, shown by the rats during the retention stage of the CFCT, with freezing response considered to provide a readout of memory that is dependent on the hippocampus, amygdala and PFC (McEchron et al. 1998; Gilmartin and Helmstetter 2010; Kochli et al. 2015). Hence, as this assessment entails cognitive/explicit and noncognitive/implicit fear memory that are supported by neural pathways that converge, the CFCT rather assesses associative memory processes by testing a memory for the association between an aversive stimulus (mild foot shock) and a salient environmental cue. Significantly enhanced freezing behavior revealed upon re-presentation of the context by one genotype rat group compared to the other would have indicated enhanced learning and memory ability. However, as our results report no difference in the frequency of freezing behavior, we conclude that this memory unit was unaffected by the genotype of the rats.

Memory as a cognitive domain can be classified according to several criteria, including function (e.g., working vs. reference memory), content (e.g. declarative/explicit vs. procedural/implicit memory), duration (e.g., immediate or short-term vs. long-term or remote memory), nature (associative vs. non-associative memory), or motivation (appetitive/reward vs. aversive memory). Hence, to allow for comprehensive profiling of mnemonic function, studies aimed at assessing differences in performance between ChAT::Cre+ versus ChAT::Cre+ rats should utilize a battery of tests that cover most, if not all fundamental types of memory processes (Savage and Ma 2014).

With evidence suggesting that the HPA-axis may be dysfunctional in children with ADHD (Hong et al. 2003), and intriguing findings indicating for a molecular interactive link between the brain's cholinergic systems and the HPA-axis neuroendocrine system in modulating cognitive processes (Paul et al. 2015), we also determined for possible defective HPA-axis activity in ChAT::Cre+ compared to Wt siblings, in response to fear conditioning. We assessed circulating levels of HPA-axis activity markers. Specifically, the stress hormone corticosterone, the main glucocorticoid in rodents (Gong et al. 2015), leptin, a $16 \mathrm{kDa}$ protein secreted by adipocytes, which has roles in controlling energy balance as well as regulating responses to stress (Haleem 2014), and glucose, since cortisol counters insulin by promoting higher blood sugar and stimulating gluconeogenesis, for synthesizing glucose (Maniam et al. 2014). Biomarkers of HPA activity could not fully explain the effects observed in the transgenic rats. In the case of corticosterone, acute stress (foot shock) induced increased plasma levels, but in both transgenic and Wt rats, to serve as a good biological validator of the stress paradigm the rats were subjected to. Exposure to the stressor did not alter levels of either leptin or glucose, with levels of these markers of HPA activity that were also independent of ChAT::Cre- genotype. It is possible that sex differences may exist in both the endocrine, cognitive behavioral and stress responses shown by the ChAT::Cre+ rats as a result of increased gene copies of VAChT, which translated to overexpressed VAChT protein levels. Future work should further investigate for such potential sex-specific differences.

The current work differentially analyzed VAChT protein and mRNA levels between the transgenic ChAT::Cre+ and non-transgenic ChAT::Cre- rats to PFC brain tissue samples. As a brain region that contains dense cholinergic innervation and outputs, the PFC was deemed to play multiple functional roles that particularly relate to the cognitive processes that the battery of behavioral tests used in the present study were designed for (Wallis 2007; Euston et al. 2012, Funahashi 2013). This includes strong arguments that have been made that ACh signaling in the PFC is strategically uniquely positioned at both the start and terminal end of the attentional loop (Sarter et al. 2001; Parikh et al. 2007). Through a series of tests, we also undertook anxiety profiling in ChAT::Cre+ versus ChAT::Cre- rats. Again, several studies implicated the PFC in anxiety-related behavior, with the PFC that was shown to exert integral control (via cholinergic reciprocal connections) over the limbic system (Kim and Whalen 2009). The strength of the connectivity between the PFC and the amygdala has further been correlated with anxiety-like behavior shown in both the OF and 
EPM (Wei et al. 2010; Delpech et al. 2016). The OF and EPM paradigms inherently also evaluate aspects of motor control, including locomotion and explorative behavior. A comprehensive body of literature implies an important role for forebrain $\mathrm{ACh}$ in controlling these motor-related aspects, as it exerts top-down control over several motor areas via reciprocal connections with the PFC (Asaad et al. 2000; Miller and Cohen 2001).

However, cholinergic neurotransmission throughout the neocortex, but also the hippocampus, amygdala and PPN can regulate arousal, learning and attention as well as aspects of emotional processing (Inglis et al. 2000, 2001). Hence, future work should aim to determine whether the presently reported molecular findings pertaining to the PFC is specific to this cholinergic brain region, or whether a similar VAChT protein and mRNA level expression pattern is evident for other brain regions containing cholinergic projection neurons in ChAT::Cre+ compared to ChAT::Cre- rats.

Taken together, the current results reveal that the ChAT::Cre rat line overexpresses 'extra' copies of a gene that functionally control cholinergic signaling. A similar effect where the BAC transgene also introduces extra copies of adjacent genes to the genome is predicted to occur in another popular transgenic rat line, namely $\mathrm{TH}: \mathrm{Cre}+$ rats (Witten et al. 2011), with such rats expressing Cre recombinase in tyrosine hydroxylase (TH) neurons. As TH acts as the rate-limiting enzyme of catecholamine biosynthesis, this rat line has, therefore, been engineered to express Cre in catecholaminergic neurons, including dopaminergic ones, that are affected progressively during neurodegenerative diseases such as PD, and has, therefore, become particularly useful for researching the pathological mechanisms concerning PD. Hence, future efforts for developing novel transgenic rat systems should utilize strategies for developing cleaner transgenic lines by eliminating unwanted genes from modified BAC DNA that could complicate phenotypic analysis.

The increasing use of genetic models, which include transgenic knockout and inbred strains of rodents has highlighted the importance of detailed characterization at behavioral and molecular-genetic levels of such rodents being used for pursuing research questions. A milestone was reached with the development of Cre/lox recombination techniques to enable conditional gene targeting in rodents. The technology utilizes $\mathrm{Cre}$ recombinase that excises a gene segment flanked by pairs of specific 34-bp DNA sequences termed LoxP recognition sequences (McLellan et al. 2017). Use of cell-specific drivers of Cre restricts recombination exclusively to cells where the driver gene is expressed, hence generating conditional knockout or knockin animals. However, the desired tissue specificity may be difficult to apply using Cre transgenic lines, with mosaic expression, leakiness of the promoter, Cre-induced toxicity, or Cre non-specificity that have been reported, all of which could confound the original purpose of the experiments. With this regard, several published reports have highlighted potential limitations posed by the use of ChAT::Cre+ mouse lines in behavioral research (e.g., Crittenden et al. 2014; Chen et al. 2018). Hence, our study provides evidence, for the first time, to strongly suggest that caution should prevail when interpreting data derived from ChAT::Cre+ rats, for functionally modulating cholinergic signaling, particularly in the utilization of cre/lox technology for evaluating the in vivo effects of the $C h A T$ gene on performance in cognitive tasks.

Acknowledgements We are grateful for highly useful suggestions made by Profs. Marco A.M. Prado, Vania F. Prado and Dr. Majid Hafezparast and also for technical advice received from Sanda Raulic and Jue Fan. We also wish to thank the following staff members for providing technical assistance in this project: Julie Gautrey, Chris Cardinal, Danilo Luise, Ali Robinson and Hayley Forest. This work was supported by a Grant from the Rosetrees Trust, awarded to ISP. CPM was supported by a Ph.D. studentship funded by Northumbria University.

\section{Compliance with ethical standards}

Conflict of interest The authors report no other biomedical financial interests or potential conflicts of interest.

Open Access This article is distributed under the terms of the Creative Commons Attribution 4.0 International License (http://creativeco mmons.org/licenses/by/4.0/), which permits unrestricted use, distribution, and reproduction in any medium, provided you give appropriate credit to the original author(s) and the source, provide a link to the Creative Commons license, and indicate if changes were made.

\section{References}

Agnoli L, Mainolfi P, Invernizzi RW, Carli M (2013) Dopamine D1-like and D2-like receptors in the dorsal striatum control different aspects of attentional performance in the five-choice serial reaction time task under a condition of increased activity of corticostriatal inputs. Neuropsychopharmacology 38:701-714

Aldrin-Kirk P, Heuer A, Rylander Ottosson D, Davidsson M, Mattsson B, Björklund T (2018) Chemogenetic modulation of cholinergic interneurons reveals their regulating role on the direct and indirect output pathways from the striatum. Neurobiol Dis 109:148-162

Alexander KS, Pocivavsek A, Wu HQ, Pershing ML, Schwarcz R, Bruno JP (2013) Early developmental elevations of brain kynurenic acid impair cognitive flexibility in adults: reversal with galantamine. Neuroscience 238:19-28

Allison C, Shoaib M (2013) Nicotine improves performance in an attentional set shifting task in rats. Neuropharmacology 64:314-320

Arrant AE, Schramm-Sapyta NL, Kuhn CM (2013) Use of the light/ dark test for anxiety in adult and adolescent male rats. Behav Brain Res 256:119-127

Asaad WF, Rainer G, Miller EK (2000) Task-specific neural activity in the primate prefrontal cortex. J Neurophysiol 84:451-459 
Azzopardi E, Louttit AG, DeOliveira C, Laviolette SR, Schmid S (2018) The role of cholinergic midbrain neurons in startle and prepulse inhibition. J Neurosci 38:8798-8808

Bari A, Dalley JW, Robbins TW (2008) The application of the 5-choice serial reaction time task for the assessment of visual attentional processes and impulse control in rats. Nat Protoc 3:759-767

Belzung C, Griebel G (2001) Measuring normal and pathological anxiety-like behaviour in mice: a review. Behav Brain Res 125:141-149

Bloem B, Poorthuis RB, Mansvelder HD (2014) Cholinergic modulation of the medial prefrontal cortex: role of nicotinic receptors in attention and regulation of neuronal activity. Front Neural Circuits 8:17

Boekhoudt L, Voets ES, Flores-Dourojeanni JP, Luijendijk MC, Vanderschuren LJ, Adan RA (2017) Chemogenetic activation of midbrain dopamine neurons affects attention, but not impulsivity, in the five-choice serial reaction time task in rats. Neuropharmacology 42:1315-1325

Bourin M, Hascoe M (2003) The mouse light/dark box test. Eur J Pharmacol 463:55-65

Bury AG, Pyle A, Elson JL, Greaves L, Morris CM, Hudson G, Pienaar IS (2017) Mitochondrial DNA changes in pedunculopontine cholinergic neurons in Parkinson's disease. Ann Neurol 82:1016-1021

Cabrera SM, Chavez CM, Corley SR, Kitto MR, Butt AE (2006) Selective lesions of the nucleus basalis magnocellularis impair cognitive flexibility. Behav Neurosci 120:298-306

Cardinal RN, Aitken MR (2010) Whisker: a client-server highperformance multimedia research control system. Behav Res Methods 42:1059-1071

Chamberlain SR, Menzies L, Hampshire A, Suckling J, Fineberg NA, Del Campo N, Aitken M, Craig K, Owen AM, Bullmore ET et al (2008) Orbitofrontal dysfunction in patients with obsessive-compulsive disorder and their unaffected relatives. Science 321:421-422

Chen E, Lallai V, Sherafat Y, Grimes NP, Pushkin AN, Fowler JP, Fowler CD (2018) Altered baseline and nicotine-mediated behavioral and cholinergic profiles in ChAT-Cre mouse lines. J Neurosci 38:2177-2188

Cohen BN, Mackey EDW, Grady SR, Mckinney S, Patzlaff NE, Wageman CR, Mcintosh JM, Marks MJ, Lester HA, Drenan RM (2012) Nicotinic cholinergic mechanisms causing elevated dopamine release and abnormal locomotor behaviour. Neuroscience 200:31-41

Cools R, Barker RA, Sahakian BJ, Robbins TW (2001) Mechanisms of cognitive set flexibility in Parkinson's disease. Brain 124:2503-2512

Crawley JN (1985) Exploratory behavior models of anxiety in mice. Neurosci Behav Rev 9:37-44

Crawley J, Goodwin FK (1980) Preliminary report of a simple animal behavior model for the anxiolytic effects of benzodiazepines. Pharmacol Biochem Behav 13:167-170

Crittenden JR, Lacey CJ, Lee T, Bowden HA, Graybiel AM (2014) Severe drug-induced repetitive behaviors and striatal overexpression of VAChT in ChAT-ChR2-EYFP BAC transgenic mice. Front Neural Circ 8:57

Croxson PL, Kyriazis DA, Baxter MG (2011) Cholinergic modulation of a specific memory function of prefrontal cortex. Nat Neurosci 14:1510-1512

Cutuli D, Foti F, Mandolesi L, De Bartolo P, Gelfo F, Federico F, Petrosini L (2009) Cognitive performances of cholinergically depleted rats following chronic donepezil administration. J Alzheimer's Dis: JAD 17:161-176

Cyr M, Parent MJ, Mechawar N, Rosa-Neto P, Soucy JP, Clark SD, Aghourian M, Bedard MA (2015) Deficit in sustained attention following selective cholinergic lesion of the pedunculopontine tegmental nucleus in rat, as measured with both post-mortem immunocytochemistry and in vivo PET imaging with $\left[{ }^{18} \mathrm{~F}\right]$ fluoroethoxybenzovesamicol. Behav Brain Res 278:107-114

Dautan D, Huerta-Ocampo I, Witten IB, Deisseroth K, Bolam JP, Gedjiikov T, Mena-Segovia J (2014) A major external source of cholinergic innervation of the striatum and nucleus accumbens originates in the brainstem. J Neurosci 34:4509-4518

Dautan D, Souza AS, Huerta-Ocampo I, Valencia M, Assous M, Witten IB, Deisseroth K, Tepper JM, Bolam JP, Gedjiikov T, MenaSegovia J (2016) Segregated cholinergic transmission modulates dopamine neurons integrated in distinct functional circuits. Nat Neurosci 19:1025-1038

Delpech JC, Wei L, Hao J, Yu X, Madore C, Butovsky O, Kaffman A (2016) Early life stress perturbs the maturation of microglia in the developing hippocampus. Brain Behav Immun 57:9-93

Eagle DM, Baunez C (2010) Is there an inhibitory-response-control system in the rat? Evidence from anatomical and pharmacological studies of behavioral inhibition. Neurosci Biobehav Rev 34:50-72

Euston DR, Gruber AJ, McNaughton BL (2012) The role of medial prefrontal cortex in memory and decision making. Neuron 76:1057-1070

Everitt BJ, Hutcheson DM, Ersche KD, Pelloux Y, Dalley JW, Robbins TW (2007) The orbital prefrontal cortex and drug addiction in laboratory animals and humans. Ann NY Acad Sci 1121:576-597

Felder CC, Goldsmith PJ, Jackson K, Sanger HE, Evans DA, Mogg AJ, Broad LM (2018) Current status of muscarinic M1 and M4 receptors as drug targets for neurodegenerative diseases. Neuropharmacology 136:449-458

Ferreira-Vieira TH, Guimaraes IM, Silva FR, Ribeiro FM (2016) Alzheimer's disease: targeting the cholinergic system. Curr Neuropharmacol 14:101-115

File SE, Gonzalez LE, Andrews N (1998) Endogenous acetylcholine in the dorsal hippocampus reduces anxiety through actions on nicotinic and muscarinic1 receptors. Behav Neurosci 112:352-359

File SE, Cheeta S, Kenny PJ (2000) Neurobiological mechanisms by which nicotine mediates different types of anxiety. Eur J Pharmacol 393:231-236

Fitzgerald PJ (2009) Neuromodulating mice and men: are there functional species differences in neurotransmitter concentration? Neurosci Biobehav Rev 33:1037-1041

Fletcher PJ, Tampakeras M, Sinyard J, Higgins GA (2007) Opposing effects of 5-HT(2A) and 5-HT(2C) receptor antagonists in the rat and mouse on premature responding in the five-choice serial reaction time test. Psychopharmacology 195:223-234

Fletcher PJ, Soko AD, Higgins GA (2013) Impulsive action in the 5 -choice serial reaction time test in 5-HT(2)c receptor null mutant mice. Psychopharmacology 226:561-570

Foster DJ, Jones CK, Conn PJ (2012) Emerging approaches for treatment of schizophrenia: modulation of cholinergic signaling. Discov Med 14:413-420

Funahashi S (2013) Thalamic mediodorsal nucleus and its participation in spatial working memory processes: comparison with the prefrontal cortex. Front Syst Neurosci 7:36

Furey ML, Drevets WC (2006) Antidepressant efficacy of the antimuscarinic drug scopolamine: a randomized, placebo-controlled clinical trial. Arch Gen Psychiatry 63:1121-1129

Gerfen CR, Paletzki R, Heintz N (2013) GENSAT BAC cre-recombinase driver lines to study the functional organization of cerebral cortical and basal ganglia circuits. Neuron 80:1368-1383

Gielow MR, Záborszky L (2017) The input-output relationship of the cholinergic basal forebrain. Cell Rep 18:1817-1830

Gilbert SJ, Burgess PW (2008) Executive function. Curr Biol 18:R110-R114 
Gilmartin MR, Helmstetter FJ (2010) Trace and contextual fear conditioning require neural activity and NMDA receptor-dependent transmission in the medial prefrontal cortex. Learn Mem 17:289-296

Giovannini MG, Rakovska A, Benton RS, Pazzagli M, Bianchi L, Pepeu G (2001) Effects of novelty and habituation on acetylcholine, GABA, and glutamate release from the frontal cortex and hippocampus of freely moving rats. Neuroscience 106:43-53

Gong S, Miao YL, Jiao GZ, Sun MJ, Li H, Lin J, Luo MJ, Tan JH (2015) Dynamics and correlation of serum cortisol and corticosterone under different physiological or stressful conditions in mice. PLoS One 10:e0117503

Gorka Z, Adamik P (1993) The effect of reserpine and stress on feeding behaviour in the light and dark phases of the diurnal cycle in rats. J Pharm Pharmacol 45:137-138

Granon S, Passetti F, Thomas KL, Dalley JW, Everitt BJ, Robbins TW (2000) Enhanced and impaired attentional performance after infusion of D1 dopaminergic receptor agents into rat prefrontal cortex. J Neurosci 20:1208-1215

Guzman MS, De Jaeger X, Raulic S, Souza IA, Li AX, Schmid S, Menon RS, Gainetdinov RR, Caron MG, Bartha R et al (2011) Elimination of the vesicular acetylcholine transporter in the striatum reveals regulation of behaviour by cholinergic-glutamatergic co-transmission. PLoS Biol 9:e1001194

Haleem DJ (2014) Investigations into the involvement of leptin in responses to stress. Behav Pharmacol 25:384-397

Heintz N (2001) BAC to the future: the use of bac transgenic mice for neuroscience research. Nat Rev Neurosci 2:861-870

Hoaglin DC, Iglewicz B (1987) Fine-tuning some resistant rules for outlier labeling. J Am Stat Assoc 82:1147-1149

Hoaglin DC, Iglewicz B, Tukey JW (1986) Performance of some resistant rules for outlier labeling. J Am Stat Assoc 81:991-999

Höhmann CF, Kwiterovich KK, Oster-Granite ML, Coyle JT (1991) Newborn basal forebrain lesions disrupt cortical cytodifferentiation as visualized by rapid Golgi staining. Cereb Cortex $1: 143-157$

Hong HJ, Shin DW, Lee EH, Oh YH, Noh KS (2003) Hypothalamicpituitary-adrenal reactivity in boys with attention deficit hyperactivity disorder. Yonsei Med J 44:608-614

Humpston CS, Wood CM, Robinson ES (2013) Investigating the roles of different monoamine transmitters and impulse control using the 5-choice serial reaction time task. J Psychopharmacol 27:213-221

Inglis WL, Dunbar JS, Winn P (2000) Pedunculopontine tegmental nucleus lesions impair stimulus-reward learning in autoshaping and conditioned reinforcement paradigms. Behav Neurosci 114:285

Inglis WL, Olmstead MC, Robbins TW (2001) Selective deficits in attentional performance on the 5-choice serial reaction time task following pedunculopontine tegmental nucleus lesions. Behav Brain Res 123:117-131

Jackson SJ, Andrews N, Ball D, Bellantuono I, Gray J, Hachoumi L, Holmes A, Latcham J, Petrie A, Potter P et al (2016) Does age matter? The impact of rodent age on study outcomes. Lab Anim 51(2):160-169

Janickova H, Prado VF, Prado MAM, El Mestikawy S, Bernard V (2017) Vesicular acetylcholine transporter (VAChT) over-expression induces major modifications of striatal cholinergic interneuron morphology and function. J Neurochem 142:857-875

Janickova H, Kljakic O, Rosborough K, Raulic S, Matovic S, Gros R, Saksida LM, Bussey TJ, Inoue W, Prado VF et al (2019) Selective decrease of cholinergic signaling from pedunculopontine and laterodorsal tegmental nuclei has little impact on cognition but markedly increases susceptibility to stress. FASEB J 33:7018-7036
Keeley RJ, Bye C, Trow J, McDonald RJ (2015) Strain and sex differences in brain and behaviour of adult rats: learning and memory, anxiety and volumetric estimates. Behav Brain Res 288:118-131

Kim JJ, Jung MW (2006) Neural circuits and mechanisms involved in Pavlovian fear conditioning: a critical review. Neurosci Biobehav Rev 30:188-202

Kim MJ, Whalen PJ (2009) The structural integrity of an amygdala-prefrontal pathway predicts trait anxiety. J Neurosci 29:1161-11618

Kochli DE, Thompson EC, Fricke EA, Postle AF, Quinn JJ (2015) The amygdala is critical for trace, delay, and contextual fear conditioning. Learn Mem 22:92-100

Koehler S, Ovadia-Caro S, van der Meer E, Villringen A, Heinz A, Romanczuk-Seiferth N, Margulies DS (2013) Increased functional connectivity between prefrontal cortex and reward system in pathological gambling. PLoS One 8:1-13

Kokare DM, Shelkar GP, Borkar CD, Nakhate KT, Subhedar NK (2011) A simple and inexpensive method to fabricate a cannula system for intracranial injections in rats and mice. J Pharmacol Toxicol Methods 64:246-250

Kolisnyk B, Guzman MS, Raulic S, Fan J, Magalhaes AC, Feng G, Gros R, Prado VF, Prado MA (2013) ChAT-ChR2-EYFP mice have enhanced motor endurance but show deficits in attention and several additional cognitive domains. J Neurosci 33:10427-10438

Labarca C, Schwarz J, Deshpande P, Schwarz S, Nowak MW, Fonck C, Nashmi R, Kofuji P, Dang H, Shi W et al (2001) Point mutant mice with hypersensitive alpha 4 nicotinic receptors show dopaminergic deficits and increased anxiety. Proc Natl Acad Sci USA 98:2786-2791

LeDoux JE (2000) Emotion circuits in the brain. Annu Rev Neurosci 23:155-184

Lezak KR, Missig G, Carlezon WA (2017) Behavioral methods to study anxiety in rodents. Dialogues Clin Neurosci 19:181-191

Livak KJ, Schmittgen TD (2001) Analysis of relative gene expression data using real-time quantitative PCR and the 2(-Delta Delta $\mathrm{C}(\mathrm{T})$ ) method. Methods 25:402-408

Madisen L, Mao T, Koch H, Zhuo JM, Berenvi A, Fujisawa S, Hsu YW, Garcia AJ, Gu X, Zanella S et al (2012) A toolbox of Credependent optogenetic transgenic mice for light-induced activation and silencing. Nat Neurosci 15:793-802

Maniam J, Antoniadis C, Morris MJ (2014) Early-life stress, HPA axis adaptation, and mechanisms contributing to later health outcomes. Front Endocrinol 5:73

Martyn AC, De Jaeger X, Magalhaes AC, Kesarwani R, Goncalves DF, Raulic S, Guzman MS, Jackson MF, Izquierdo I, Macdonals JF et al (2012) Elimination of the vesicular acetylcholine transporter in the forebrain causes hyperactivity and deficits in spatial memory and long-term potentiation. Proc Natl Acad Sci USA 109:17651-17656

McCutcheon JE, Marinelli M (2009) Age matters. Eur J Neurosci 29:997-1014

McEchron MD, Bouwmeester H, Tseng W, Weiss C, Disterhoft JF (1998) Hippocampectomy disrupts auditory trace fear conditioning and contextual fear conditioning in the rat. Hippocampus 8:638-646

McGaughy J, Dalley JW, Morrison CH, Everitt BJ, Robbins TW (2002) Lesions produced by intrabasalis infusions of $192 \mathrm{IgG}$-Saporin on attentional performance in a five-choice serial reaction time task. Neuroscience 22:1905-1913

McGaughy J, Ross RS, Eichenbaum H (2008) Noradrenergic, but not cholinergic, deafferentation of prefrontal cortex impairs attentional set-shifting. Neuroscience 153:63-71

McLellan MA, Rosenthal NA, Pinto AR (2017) Cre-loxP-mediated recombination: general principles and experimental considerations. Curr Protoc Mouse Biol 7:1-12 
Mesulam MM, Mufson EJ, Levey AI, Wainer BH (1984) Atlas of cholinergic neurons in the forebrain and upper brainstem of the macaque based on monoclonal choline acetyltransferase immunohistochemistry and acetylcholinesterase histochemistry. Neuroscience 12:669-686

Miller EK, Cohen JD (2001) An integrative theory of prefrontal cortex function. Annu Rev Neurosci 24:167-202

Mineur YS, Obayemi A, Wigestrand MB, Fote GM, Calarco CA, Li AM, Picciotto MR (2013) Cholinergic signaling in the hippocampus regulates social stress resilience and anxiety- and depressionlike behavior. Proc Natl Acad Sci USA 110:3573-3578

Mizuno T, Endo Y, Arita J, Kimura F (1991) Acetylcholine release in the rat hippocampus as measured by the microdialysis method correlates with motor activity and exhibits a diurnal variation. Neuroscience 44:607-612

Nagy PM, Aubert I (2012) Overexpression of the vesicular acetylcholine transporter increased acetylcholine release in the hippocampus. Neuroscience 218:1-11

Nagy PM, Aubert I (2013) B6eGFPChAT mice overexpressing the vesicular acetylcholine transporter exhibit spontaneous hypoactivity and enhanced exploration in novel environments. Brain Behav 3:367-383

Nagy PM, Aubert I (2015) Overexpression of the vesicular acetylcholine transporter enhances dendritic complexity of adult-born hippocampal neurons and improves acquisition of spatial memory during aging. Neurobiol Aging 36:1881-1889

Newman MB, Nazian SJ, Sanberg PR, Diamond DM, Shytle RD (2001) Corticosterone-attenuating and anxiolytic properties of mecamylamine in the rat. Prog Neuropsychopharmacol Biol Psychiatry 25:609-620

Nikiforuk A, Kos T, Potasiewicz A, Popik P (2015) Positive allosteric modulation of alpha 7 nicotinic acetylcholine receptors enhances recognition memory and cognitive flexibility in rats. Eur Neuropsychopharmacol 25:1300-1313

Parikh V, Kozak R, Martinez V, Sarter M (2007) Prefrontal acetylcholine release controls cue detection on multiple timescales. Neuron 56:141-154

Patton JH, Stanford MS, Barratt ES (1995) Factor structure of the Barratt impulsiveness scale. J Clin Psychol 51:768-774

Paul S, Jeon WK, Bizon JL, Han JS (2015) Interaction of basal forebrain cholinergic neurons with the glucocorticoid system in stress regulation and cognitive impairment. Front Aging Neurosci 4:43

Perez-Lloret S, Barrantes FJ (2016) Deficits in cholinergic neurotransmission and their clinical correlates in Parkinson's disease. NPJ Parkinsons Dis 2:16001

Pienaar IS, Gartside SE, Sharma P, De Paola V, Gretenkord S, Withers S, Elson JL, Dexter DT (2015) Pharmacogenetic stimulation of cholinergic pedunculopontine neurons reverses motor deficits in a rat model of Parkinson's disease. Mol Neurodegener 10:47

Podhorna J, Franklin KB (1999) Long-lasting increase in anxiety after electrolytic lesions of the pedunculopontine tegmental nucleus. Behav Neurosci 113:550-557

Podhorna J, Franklin KB (2000) Pontine tegmentum lesions increase anxiety-like behavior in rats: a comparison with anxiety produced by beta-CCE. Pharmacol Biochem Behav 65:267-273

Prado VF, Martins-Silva C, de Castro BM, Lima RF, Barros DM, Amaral E, Ramsey AJ, Sotnikova TD, Ramirez MR, Kim HG et al (2006) Mice deficient for the vesicular acetylcholine transporter are myasthenic and have deficits in object and social recognition. Neuron 51:601-612

Prado VF, Roy A, Kolisnyk B, Gros R, Prado MAM (2013) Regulation of cholinergic activity by the vesicular acetylcholine transporter. Biochem J 450:265-274

Prado VF, Janickova H, Al-Onaizi MA, Prado MA (2017) Cholinergic circuits in cognitive flexibility. Neuroscience 345:130-141
Prut L, Belzung C (2003) The open field as a paradigm to measure the effects of drugs on anxiety-like behaviors: a review. Eur J Pharmacol 463:3-33

Pugh CR, Tremblay D, Fleshner M, Rudy JW (1997) A selective role for corticosterone in contextual-fear conditioning. Behav Neurosci 111:503-511

Rice ME, Cragg SJ (2004) Nicotine amplifies reward-related dopamine signals in striatum. Nat Neurosci 7:583-584

Ridley RM, Baker JA, Baker HF, Maclean CJ (1994) Restoration of cognitive abilities by cholinergic grafts in cortex of monkeys with lesions of the basal nucleus of Meynert. Neuroscience 63:653-666

Robbins TW (2002) The 5-choice serial reaction time task: behavioural pharmacology and functional neurochemistry. Psychopharmacology $163: 362-380$

Rubia K, Smith AB, Halari R, Matsukura F, Mohammad M, Taylor E, Brammer MJ (2009) Disorder-specific dissociation of orbitofrontal dysfunction in boys with pure conduct disorder during reward and ventrolateral prefrontal dysfunction in boys with pure ADHD during sustained attention. Am J Psychiatry 166:83-94

Salamone JD, Correa M (2012) The mysterious motivational functions of mesolimbic dopamine. Neuron 76:470-485

Saricicek A, Esterlis I, Maloney KH, Mineur YS, Ruf BM, Muralidharan A, Chen JI, Cosgrove KP, Kerestes R, Ghose S et al (2012) Persistent $\beta 2 *$-nicotinic acetylcholinergic receptor dysfunction in major depressive disorder. Am J Psychiatry 169:851-859

Sarter M, Givens B, Bruno JP (2001) The cognitive neuroscience of sustained attention: where top-down meets bottom-up. Brain Res Brain Res Rev 35:146-160

Sarter M, Lustig C, Taylor SF (2012) Cholinergic contributions to the cognitive symptoms of schizophrenia and the viability of cholinergic treatments. Neuropharmacology 62:1544-1553

Savage S, Ma D (2014) III. Animal behaviour testing: memory. Br J Anaseth 113:6-9

Semenova S, Stolerman IP, Markou A (2007) Chronic nicotine administration improves attention while nicotine withdrawal induces performance deficits in the 5-choice serial reaction time task in rats. Pharmacol Biochem Behav 87:360-368

Sestakova N, Puzserova A, Kluknavsky M, Bernatova I (2013) Determination of motor activity and anxiety-related behaviour in rodents: methodological aspects and role of nitric oxide. Interdiscip Toxicol 6:126-135

Sharma PK, Pienaar IS (2014) Pharmacogenetic and optical dissection for mechanistic understanding of Parkinson's disease: potential utilities revealed through behavioural assessment. Neurosci Biobehav Rev 47:87-100

Sharma PK, Pienaar IS (2018) The use of DREADDs for dissecting the contribution of neural circuit mechanisms in models of neurodegenerative disease. In: Crusio WE, Gerlai RT (eds) Molecular-genetic and statistical techniques for behavioral and neural research. Elsevier, New York, pp 565-596

Shoaib M, Bizarro L (2005) Deficits in a sustained attention task following nicotine withdrawal in rats. Psychopharmacology 178:211-222

Snyder JS, Choe JS, Clifford MA, Jeurling SI, Hurley P, Brown A, Kambi JF, Cameron HA (2009) Adult-born hippocampal neurons are more numerous, faster maturing, and more involved in behavior in rats than in mice. J Neurosci 29:14484-14495

Sugita S, Fleming LL, Wood C, Vaughan SK, Gomes MP, Camargo W, Naves LA, Prado VF, Prado MA, Guatimosim C et al (2016) VAChT overexpression increases acetylcholine at the synaptic cleft and accelerates aging of neuromuscular junctions. Skelet Muscle 6:31

Tait DS, Brown VJ (2008) Lesions of the basal forebrain impair reversal learning but not shifting of attentional set in rats. Behav Brain Res 187:100-108 
Tait DS, Chase EA, Brown VJ (2013) Tacrine improves reversal learning in older rats. Neuropharmacology 73:284-289

Tallini YN, Shui B, Greene KS, Deng KY, Doran R, Fisher PJ, Zipfel W, Kotlikoff MI (2006) BAC transgenic mice express enhanced green fluorescent protein in central and peripheral cholinergic neurons. Physiol Genomics 27:391-397

Taniguchi H, He M, Wu P, Kim S, Paik R, Sugino K, Kvitsiani D, Fu Y, Lu J, Lin Y et al (2011) A resource of Cre driver lines for genetic targeting of GABAergic neurons in cerebral cortex. Neuron 71:995-1013

Tavote P, Fadok JP, Luthi A (2015) Neural circuits for fear and anxiety. Nat Rev Neurosci 16:317-331

Thiel CM, Huston JP, Schwarting RK (1998) Hippocampal acetylcholine and habituation learning. Neuroscience 85:1253-1262

Thompson BL, Erickson K, Schulkin J, Rosen JB (2004) Corticosterone facilitates retention of contextually conditioned fear and increases CRH mRNA expression in the amygdala. Behav Brain Res 149:209-215

Tingley D, Alexander AS, Quinn LK, Chiba AA, Nitz DA (2015) Cell assemblies of the basal forebrain. J Neurosci 35:2992-3000

Walf AA, Frye CA (2007) The use of the elevated plus maze as an assay of anxiety-related behavior in rodents. Nat Protoc 2:322-328
Wallis JD (2007) Orbitofrontal cortex and its contribution to decisionmaking. Annu Rev Neurosci 30:31-56

Wei L, David A, Duman RS, Anisman H, Kaffman A (2010) Early life stress increases anxiety-like behavior in Balb c mice despite a compensatory increase in levels of postnatal maternal care. Horm Behav 57:396-404

Witten IB, Steinberg EE, Lee SY, Davidson TJ, Zalocusky KA, Brodsky M, Yizhar O, Cho SL, Gong S, Ramakrishnan C et al (2011) Recombinase-driver rat lines: tools, techniques, and optogenetic application to dopamine-mediated reinforcement. Neuron 72:721-733

Xiao C, Cho JR, Zhou C, Treweek JB, Chan K, McKinney SL, Yan B, Gradinaru V (2016) Cholinergic mesopontine signals govern locomotion and reward through dissociable midbrain pathways. Neuron 90:333-347

Publisher's Note Springer Nature remains neutral with regard to jurisdictional claims in published maps and institutional affiliations. 